\title{
On the Grassmann modules for the unitary groups
}

\author{
Bart De Bruyn \\ Ghent University, Department of Pure Mathematics and Computer Algebra, \\ Krijgslaan 281 (S22), B-9000 Gent, Belgium, E-mail: bdb@cage.ugent. be
}

\begin{abstract}
Let $V$ be $2 n$-dimensional vector space over a field $\mathbb{K}$ equipped with a nondegenerate skew- $\psi$-Hermitian form $f$ of Witt index $n \geq 1$, let $\mathbb{K}_{0} \subseteq \mathbb{K}$ be the fix field of $\psi$ and let $G$ denote the group of isometries of $(V, f)$. For every $k \in\{1, \ldots, 2 n\}$, there exist natural representations of the groups $G \cong U\left(2 n, \mathbb{K} / \mathbb{K}_{0}\right)$ and $H=G \cap S L(V) \cong S U\left(2 n, \mathbb{K} / \mathbb{K}_{0}\right)$ on the $k$-th exterior power of $V$. With the aid of linear algebra, we prove some properties of these representations. We also discuss some applications to projective embeddings and hyperplanes of Hermitian dual polar spaces.
\end{abstract}

Keywords: Grassmann module, unitary group, Hermitian dual polar space, hyperplane

MSC2000: 15A75, 15A63, 20C33, 51A50

\section{Introduction}

This paper is an essay in which we will use methods based on linear algebra to derive several facts regarding structures which are related to a $2 n$-dimensional $\mathbb{K}$-vector space $V$ which is endowed with a nondegenerate skew-Hermitian form $f$ of maximal Witt index $n$. These methods allow us to give more elegant proofs for some known results, and to state some known results in a language which is more elegant and more suitable for future applications. More precisely, we will do the following:

(1) If $\psi$ denotes the involutary automorphism of $\mathbb{K}$ associated to $f$ and if $\mathbb{K}_{0} \subset \mathbb{K}$ denotes the fix field of $\psi$, then we will prove the irreducibility of certain modules for the groups $U\left(2 n, \mathbb{K} / \mathbb{K}_{0}\right)$ and $S U\left(2 n, \mathbb{K} / \mathbb{K}_{0}\right)$. 
(2) We will give a more elegant description (and a more elegant proof for the existence) of the Baer- $\mathbb{K}_{0}$-subgeometry $\operatorname{PG}\left(W^{*}\right)$ of $\operatorname{PG}\left(\bigwedge^{n} V\right)$ which affords the Grassmann embedding of the dual polar space $D H(2 n-1, \mathbb{K}, \psi)$ associated to $(V, f)$.

(3) Every hyperplane $\mathcal{H}$ of $D H(2 n-1, \mathbb{K}, \psi)$ which arises from the Grassmann embedding can be described by a certain vector of $W^{*}$, a so-called representative vector of $\mathcal{H}$. De Bruyn and Pralle [9] proved that the finite Hermitian dual polar space $D H\left(5, q^{2}\right)$ has 5 isomorphism classes of hyperplanes arising from the Grassmann embedding. We determine a representative vector for each of these 5 isomorphism classes.

Remark. In [8], we used techniques based on linear algebra to derive several facts regarding structures related to a $2 n$-dimensional vector space endowed with a nondegenerate alternating bilinear form.

\subsection{Certain representations of unitary groups}

Let $n$ be a strictly positive integer and let $\mathbb{K}_{0}, \mathbb{K}$ be two fields such that $\mathbb{K}$ is a quadratic Galois extension of $\mathbb{K}_{0}$. Put $\mathbb{K}^{*}:=\mathbb{K} \backslash\{0\}$ and $\mathbb{K}_{0}^{*}:=\mathbb{K}_{0} \backslash\{0\}$. Let $\psi$ denote the unique nontrivial element in $\operatorname{Gal}\left(\mathbb{K} / \mathbb{K}_{0}\right)$ and let $V$ be a $2 n$-dimensional vector space over $\mathbb{K}$ equipped with a nondegenerate skew- $\psi$ Hermitian form $f$ of Witt index $n$.

An ordered basis $\left(\bar{e}_{1}, \bar{f}_{1}, \ldots, \bar{e}_{n}, \bar{f}_{n}\right)$ of $V$ is called a hyperbolic basis of $V$ if $f\left(\bar{e}_{i}, \bar{e}_{j}\right)=f\left(\bar{f}_{i}, \bar{f}_{j}\right)=0$ and $f\left(\bar{e}_{i}, \bar{f}_{j}\right)=\delta_{i j}$ for all $i, j \in\{1, \ldots, n\}$. Let $G$ denote the group of isometries of $(V, f)$, i.e. the set of all $\theta \in G L(V)$ satisfying $f(\theta(\bar{x}), \theta(\bar{y}))=f(\bar{x}, \bar{y})$ for all $\bar{x}, \bar{y} \in V$. Then $G \cong U\left(2 n, \mathbb{K} / \mathbb{K}_{0}\right)$ and $H:=G \cap S L(V) \cong S U\left(2 n, \mathbb{K} / \mathbb{K}_{0}\right)$. The elements of $G$ are precisely those elements of $G L(V)$ which map hyperbolic bases of $V$ to hyperbolic bases of $V$. It can be proved (see Lemma 2.2) that if $\theta \in G$, then there exists an $\eta \in \mathbb{K}^{*}$ such that $\operatorname{det}(\theta)=\frac{\eta^{\psi}}{\eta}$. We denote by $\eta_{\theta}$ any of the elements of $\mathbb{K}^{*}$ satisfying this property. The element $\eta_{\theta}$ is uniquely determined up to a factor of $\mathbb{K}_{0}^{*}$. If $\theta_{1}, \theta_{2} \in G$, then $\eta_{\theta_{2} \circ \theta_{1}} \cdot \eta_{\theta_{1}}^{-1} \cdot \eta_{\theta_{2}}^{-1} \in \mathbb{K}_{0} \operatorname{since} \operatorname{det}\left(\theta_{2} \circ \theta_{1}\right)=\operatorname{det}\left(\theta_{1}\right) \cdot \operatorname{det}\left(\theta_{2}\right)$.

For every $k \in\{0, \ldots, 2 n\}$, let $\bigwedge^{k} V$ be the $k$-th exterior power of $V$. Then $\bigwedge^{0} V=\mathbb{K}$ and $\bigwedge^{1} V=V$. If $k \in\{1, \ldots, 2 n\}$, then for every $\theta \in G L(V)$, there exists a unique $\widetilde{\theta}_{k} \in G L\left(\bigwedge^{k} V\right)$ such that $\widetilde{\theta}_{k}\left(\bar{v}_{1} \wedge \bar{v}_{2} \wedge \cdots \wedge \bar{v}_{k}\right)=$ $\theta\left(\bar{v}_{1}\right) \wedge \theta\left(\bar{v}_{2}\right) \wedge \cdots \wedge \theta\left(\bar{v}_{k}\right)$ for all vectors $\bar{v}_{1}, \bar{v}_{2}, \ldots, \bar{v}_{k} \in V$. The map $\theta \mapsto \widetilde{\theta}_{k}$ define representations $\mathcal{R}_{k}$ and $\mathcal{R}_{k}^{\prime}$ of the respective groups $G \cong U\left(2 n, \mathbb{K} / \mathbb{K}_{0}\right)$ and $H \cong S U\left(2 n, \mathbb{K} / \mathbb{K}_{0}\right)$ on the $\left(\begin{array}{c}2 n \\ k\end{array}\right)$-dimensional vector space $\bigwedge^{k} V$. We call the corresponding $\mathbb{K} G$-modules (respectively $\mathbb{K} H$-modules) Grassmann modules for $G$ (respectively $H$ ). We put $\widetilde{G}_{k}:=\left\{\widetilde{\theta}_{k} \mid \theta \in G\right\}$ and $\widetilde{H}_{k}:=$ 
$\left\{\widetilde{\theta}_{k} \mid \theta \in H\right\}$. The following result might be known (during the course of writing this paper, the author observed that a group-theoretical proof of this fact is also contained in the preprint [2]). Anyhow, we will prove it in Section 3 with the aid of elementary linear algebra.

Theorem 1.1 For every $k \in\{1, \ldots, 2 n\}$, the representation $\mathcal{R}_{k}^{\prime}$ is irreducible.

Theorem 1.1 has the following corollary:

Corollary 1.2 (1) For every $k \in\{1, \ldots, 2 n\}$, the representation $\mathcal{R}_{k}$ is irreducible.

(2) For every $k \in\{1, \ldots, n\}$, the subspace of $\bigwedge^{k} V$ generated by all vectors of the form $\bar{v}_{1} \wedge \cdots \wedge \bar{v}_{k}$ such that $\left\langle\bar{v}_{1}, \ldots, \bar{v}_{k}\right\rangle$ is totally isotropic with respect to $f$ coincides with $\bigwedge^{k} V$.

Proof. Claim (1) follows from the fact that $H$ is a subgroup of $G$.

Now, let $k \in\{1, \ldots, n\}$. Obviously, the subspace of $\bigwedge^{k} V$ generated by all vectors of the form $\bar{v}_{1} \wedge \cdots \wedge \bar{v}_{k}$ such that $\left\langle\bar{v}_{1}, \ldots, \bar{v}_{k}\right\rangle$ is totally isotropic with respect to $f$ is stabilized by $\widetilde{G}_{k}$. Claim (2) then follows from Claim (1).

In Section 4, we prove the following:

Theorem 1.3 There exists a set $W^{*}$ of vectors of $\bigwedge^{n} V$ satisfying the following properties:

(1) The set $W^{*}$ is a $\left(\begin{array}{c}2 n \\ n\end{array}\right)$-dimensional vector space over $\mathbb{K}_{0}$ (with addition of vectors and multiplication with scalars inherited from $\bigwedge^{n} V$ ).

(2) For every $\theta \in G, \widetilde{\theta}_{n}\left(W^{*}\right)=\left\{\frac{\alpha}{\eta_{\theta}} \mid \alpha \in W^{*}\right\}$.

If $\theta \in H$, then $\eta_{\theta} \in \mathbb{K}_{0}^{*}$ and we have

Corollary 1.4 If $\theta \in H$, then $\widetilde{\theta}_{n}\left(W^{*}\right)=W^{*}$.

Now, for every map $\theta \in H$, let $\widehat{\theta}$ be the element of $G L\left(W^{*}\right)$ mapping $\alpha \in W^{*}$ to $\widetilde{\theta}_{n}(\alpha) \in W^{*}$. Then the map $\theta \mapsto \widehat{\theta}$ defines a representation $\widehat{\mathcal{R}}$ of the group $H \cong S U\left(2 n, \mathbb{K} / \mathbb{K}_{0}\right)$ on the $\left(\begin{array}{c}2 n \\ n\end{array}\right)$-dimensional $\mathbb{K}_{0^{-}}$-vector space $W^{*}$. The corresponding $\mathbb{K}_{0} H$-module is also called a Grassmann module for $S U\left(2 n, \mathbb{K} / \mathbb{K}_{0}\right)$. Put $\widehat{H}:=\{\widehat{\theta} \mid \theta \in H\}$. As a consequence of Theorem 1.1, we have

Corollary 1.5 The representation $\widehat{\mathcal{R}}$ is irreducible. 
Proof. Suppose $U$ is a subspace of $W^{*}$ which is stabilized by $\widehat{H}$. The subspace $U$ is contained in a unique subspace $\bar{U}$ of $\bigwedge^{n} V$ with the same dimension as $U$. Obviously, $\bar{U}$ is stabilized by $\widetilde{H}_{n}$. So by Theorem 1.1, either $\bar{U}=0$ or $\bar{U}=\bigwedge^{n} V$. Hence, either $U=0$ or $U=W^{*}$.

\subsection{The Grassmann embedding of the dual polar space $D H(2 n-1, \mathbb{K}, \psi)$}

A full (projective) embedding of a point-line geometry $\mathcal{S}$ is an injective mapping $e$ from the point-set $\mathcal{P}$ of $\mathcal{S}$ to the point-set of a projective space $\Sigma$ satisfying (i) $\langle e(\mathcal{P})\rangle_{\Sigma}=\Sigma$ and (ii) $e(L)$ is a line of $\Sigma$ for every line $L$ of $\mathcal{S}$.

Let $\Pi$ be a polar space (Tits [12], Veldkamp [13]) of rank $n \geq 2$. With $\Pi$ there is associated a point-line geometry $\Delta$ which is called a dual polar space, see Cameron [3]. The points of $\Delta$ are the maximal singular subspaces of $\Pi$, the lines of $\Delta$ are the next-to-maximal singular subspaces of $\Pi$, and incidence is reverse containment. If $\omega_{1}$ and $\omega_{2}$ are two maximal singular subspaces of $\Pi$, then $\mathrm{d}\left(\omega_{1}, \omega_{2}\right)$ denotes the distance between $\omega_{1}$ and $\omega_{2}$ in the collinearity graph of $\Delta$. We have $\mathrm{d}\left(\omega_{1}, \omega_{2}\right)=n-1-\operatorname{dim}\left(\omega_{1} \cap \omega_{2}\right)$. The points $\omega_{1}$ and $\omega_{2}$ of $\Delta$ are called opposite if they lie at maximal distance $n$ from each other. The dual polar space $\Delta$ is a near polygon, which means that for every point $x$ and every line $L$ there exists a unique point on $L$ nearest to $x$. If $x$ is a point of $\Delta$, then $x^{\perp}$ denotes the set of points of $\Delta$ equal to or collinear with $x$. There exists a bijective correspondence between the possibly empty singular subspaces of $\Pi$ and the nonempty convex subspaces of $\Delta$. If $\omega$ is an $(n-1-k)$-dimensional singular subspace of $\Pi$, then the set of all maximal singular subspaces of $\Pi$ containing $\omega$ is a convex subspace of $\Delta$ of diameter $k$. These convex subspaces are called quads if $k=2$. Any two points $x_{1}$ and $x_{2}$ of $\Delta$ at distance $k$ from each other are contained in a unique convex subspace $\left\langle x_{1}, x_{2}\right\rangle$ of diameter $k$. If $x$ is a point and $S$ is a convex subspace, then there exists a unique point $\pi_{S}(x) \in S$ such that $\mathrm{d}(x, y)=\mathrm{d}\left(x, \pi_{S}(x)\right)+\mathrm{d}\left(\pi_{S}(x), y\right)$ for every point $y \in S$. The convex subspaces through a given point $x$ of $\Delta$ define an $(n-1)$-dimensional projective space which we will denote by $\operatorname{Res}(x)$.

As in Section 1.1, let $V$ be a $2 n$-dimensional vector space over $\mathbb{K}$ equipped with a nondegenerate skew- $\psi$-Hermitian form $f$ of Witt index $n \geq 2$. With the nondegenerate skew- $\psi$-Hermitian form $f$, there is associated a Hermitian polar space $H(2 n-1, \mathbb{K}, \psi)$ and a Hermitian dual polar space $D H(2 n-$ $1, \mathbb{K}, \psi)$. The singular subspaces of $H(2 n-1, \mathbb{K}, \psi)$ are the subspaces of $\mathrm{PG}(2 n-1, \mathbb{K})$ which are totally isotropic with respect to the Hermitian 
polarity of $P G(V)$ defined by $f$. In Section 4, we will prove the following regarding the vector space $W^{*}$ alluded to in Theorem 1.3.

Theorem 1.6 (1) For every maximal singular subspace $\omega=\left\langle\bar{v}_{1}, \bar{v}_{2}, \ldots, \bar{v}_{n}\right\rangle$ of $H(2 n-1, \mathbb{K}, \psi)$, there exists a unique point $e_{g r}(\omega)=\langle\beta\rangle$ in $\mathrm{PG}\left(W^{*}\right)$ such that $\beta \in W^{*}$ and $\bar{v}_{1} \wedge \bar{v}_{2} \wedge \cdots \wedge \bar{v}_{n}$ are linearly dependent vectors of $\wedge^{n} V$.

(2) The map $\omega \mapsto e_{g r}(\omega)$ defines a full embedding of $D H(2 n-1, \mathbb{K}, \psi)$ into the Baer- $\mathbb{K}_{0}$-subgeometry $\mathrm{PG}\left(W^{*}\right)$ of $\mathrm{PG}\left(\bigwedge^{n} V\right)$.

The projective embedding $e_{g r}$ mentioned in Theorem 1.6(2) is called the Grassmann embedding of $D H(2 n-1, \mathbb{K}, \psi)$.

Remark. Another description of the Baer- $\mathbb{K}_{0}$-subgeometry of $\operatorname{PG}\left(\bigwedge^{n} V\right)$ which affords the Grassmann embedding of $D H(2 n-1, \mathbb{K}, \psi)$ was given in [7]. The description and proof which we will give in Section 4 seem more elegant. In [5, Proposition 5.1], there was given a description of a $\mathbb{K}_{0}$-vector space $W \subseteq \bigwedge^{n} V$ stabilized by $\widetilde{H}_{n}$ such that $\mathrm{PG}(W)$ affords the Grassmann embedding of $D H(2 n-1, \mathbb{K}, \psi)$. The proof given in [5] is however not correct as was already pointed out in [7]. Also some corrections must be performed in [5] in order to get the right equation for $W$ (e.g., observe the coefficient $(-1)^{l}$ in the formula at the beginning of Section 4).

A set of points of $D H(2 n-1, \mathbb{K}, \psi)$ distinct from the whole point-set is called a hyperplane of $D H(2 n-1, \mathbb{K}, \psi)$ if it intersects every line in either a singleton or the whole line. If $\pi$ is a hyperplane of $\mathrm{PG}\left(W^{*}\right)$, then the set of all points $p$ of $D H(2 n-1, \mathbb{K}, \psi)$ such that $e_{g r}(p) \in \pi$ is a hyperplane of $D H(2 n-1, \mathbb{K}, \psi)$. Any hyperplane of $D H(2 n-1, \mathbb{K}, \psi)$ which can be obtained in this way is said to arise from $e_{g r}$.

If $\mathbb{K}$ is the finite field $\mathbb{F}_{q^{2}}$ with $q^{2}$ elements (so, $\mathbb{K}_{0} \cong \mathbb{F}_{q}$ and $\psi: \mathbb{K} \rightarrow \mathbb{K}$ : $\left.x \mapsto x^{q}\right)$, then we will denote $H(2 n-1, \mathbb{K}, \psi)$ and $D H(2 n-1, \mathbb{K}, \psi)$ also by $H\left(2 n-1, q^{2}\right)$ and $D H\left(2 n-1, q^{2}\right)$.

Consider now the special case $n=3, \mathbb{K}=\mathbb{F}_{q^{2}}$ and let $\mathcal{A}$ denote the group of automorphisms of $D H\left(5, q^{2}\right)$. For every $\varphi \in \mathcal{A}$, there exists a unique collineation $\widetilde{\varphi}$ of $\operatorname{PG}\left(W^{*}\right)$ such that $e_{g r}(\varphi(p))=\widetilde{\varphi}\left(e_{g r}(p)\right)$ for every point $p$ of $\operatorname{DH}\left(5, q^{2}\right)$. By De Bruyn and Pralle [9], the group $\mathcal{A}$ has 5 orbits on the set of hyperplanes of $D H\left(5, q^{2}\right)$ arising from $e_{g r}$. In Section 5, we will show that this implies that $\widetilde{A}:=\{\widetilde{\varphi} \mid \varphi \in \mathcal{A}\}$ has 5 orbits on the set of points of $\mathrm{PG}\left(W^{*}\right)$, and we will determine an explicit description of a point of each of these five orbits. 


\section{Hyperbolic bases of $V$}

In this section, we continue with the notation introduced in Section 1.1. Recall that $V$ is a $2 n$-dimensional vector space $(n \geq 1)$ over $\mathbb{K}$ which is equipped with a nondegenerate skew- $\psi$-Hermitian form $f$ of Witt index $n$, and that $\mathbb{K}_{0}$ is the fix field of $\psi$.

If $\left(\bar{e}_{1}, \bar{f}_{1}, \ldots, \bar{e}_{n}, \bar{f}_{n}\right)$ is a hyperbolic basis of $V$, then

(1) for every permutation $\sigma$ of $\{1, \ldots, n\}$, also $\left(\bar{e}_{\sigma(1)}, \bar{f}_{\sigma(1)}, \ldots, \bar{e}_{\sigma(n)}, \bar{f}_{\sigma(n)}\right)$ is a hyperbolic basis of $V$;

(2) for every $\lambda \in \mathbb{K}^{*}$, also $\left(\frac{\bar{e}_{1}}{\lambda}, \lambda^{\psi} \bar{f}_{1}, \bar{e}_{2}, \bar{f}_{2}, \ldots, \bar{e}_{n}, \bar{f}_{n}\right)$ is a hyperbolic basis of $V$;

(3) for every $\lambda \in \mathbb{K}$, also $\left(\bar{e}_{1}+\lambda \bar{e}_{2}, \bar{f}_{1}, \bar{e}_{2},-\lambda^{\psi} \bar{f}_{1}+\bar{f}_{2}, \bar{e}_{3}, \bar{f}_{3}, \ldots, \bar{e}_{n}, \bar{f}_{n}\right)$ is a hyperbolic basis of $V$;

(4) for every $\lambda \in \mathbb{K}_{0}$, also $\left(\bar{e}_{1}, \bar{f}_{1}, \ldots, \bar{e}_{n-1}, \bar{f}_{n-1}, \bar{e}_{n}, \bar{f}_{n}+\lambda \bar{e}_{n}\right)$ is a hyperbolic basis of $V$;

(5) for every $\lambda \in \mathbb{K}_{0}$, also $\left(\bar{e}_{1}, \bar{f}_{1}, \ldots, \bar{e}_{n-1}, \bar{f}_{n-1}, \bar{e}_{n}+\lambda \bar{f}_{n}, \bar{f}_{n}\right)$ is a hyperbolic basis of $V$.

For every $i \in\{1,2,3,4,5\}$, let $\Omega_{i}$ denote the set of all ordered pairs $\left(B_{1}, B_{2}\right)$ of hyperbolic bases of $V$ such that $B_{2}$ can be obtained from $B_{1}$ as described in $(i)$ above.

Lemma 2.1 If $B$ and $B^{\prime}$ are two hyperbolic bases of $V$, then there exist hyperbolic bases $B_{0}, B_{1}, \ldots, B_{k}(k \geq 0)$ of $V$ such that $B_{0}=B, B_{k}=B^{\prime}$ and $\left(B_{i-1}, B_{i}\right) \in \Omega_{1} \cup \cdots \cup \Omega_{5}$ for every $i \in\{1, \ldots, k\}$.

Proof. Put $B=\left(\bar{e}_{1}, \bar{f}_{1}, \ldots, \bar{e}_{n}, \bar{f}_{n}\right)$ and $B^{\prime}=\left(\bar{e}_{1}^{\prime}, \bar{f}_{1}^{\prime}, \ldots, \bar{e}_{n}^{\prime}, \bar{f}_{n}^{\prime}\right)$. Put $E=$ $\left\langle\bar{e}_{1}, \ldots, \bar{e}_{n}\right\rangle, E^{\prime}=\left\langle\bar{e}_{1}^{\prime}, \ldots, \bar{e}_{n}^{\prime}\right\rangle, F=\left\langle\bar{f}_{1}, \ldots, \bar{f}_{n}\right\rangle$ and $F^{\prime}=\left\langle\bar{f}_{1}^{\prime}, \ldots, \bar{f}_{n}^{\prime}\right\rangle$. The proof of the lemma will occur in 3 steps.

(1) Suppose $E=E^{\prime}$ and $F=F^{\prime}$. Since the maps $\left(\bar{g}_{1}, \bar{g}_{2}, \ldots, \bar{g}_{n}\right) \mapsto$ $\left(\bar{g}_{\sigma(1)}, \bar{g}_{\sigma(2)}, \ldots, \bar{g}_{\sigma(n)}\right),\left(\bar{g}_{1}, \bar{g}_{2}, \ldots, \bar{g}_{n}\right) \mapsto\left(\frac{\bar{g}_{1}}{\lambda}, \bar{g}_{2}, \ldots, \bar{g}_{n}\right)$ and $\left(\bar{g}_{1}, \bar{g}_{2}, \ldots, \bar{g}_{n}\right) \mapsto$ $\left(\bar{g}_{1}+\lambda \bar{g}_{2}, \bar{g}_{2}, \ldots, \bar{g}_{n}\right)$ allow us to transform any basis of $E$ to any other basis of $E$, there exist hyperbolic bases $B_{0}, B_{1}, \ldots, B_{k}(k \geq 0)$ of $V$ such that (i) $B_{0}=B$, (ii) $\left(B_{i-1}, B_{i}\right) \in \Omega_{1} \cup \Omega_{2} \cup \Omega_{3}$ for every $i \in\{1, \ldots, k\}$, and (iii) $B_{k}$ is of the form $\left(\bar{e}_{1}^{\prime}, \bar{f}_{1}^{\prime \prime}, \ldots, \bar{e}_{n}^{\prime}, \bar{f}_{n}^{\prime \prime}\right)$ with $F=\left\langle\bar{f}_{1}^{\prime \prime}, \ldots, \bar{f}_{n}^{\prime \prime}\right\rangle$. The vector $\bar{f}_{i}^{\prime \prime}$, $i \in\{1, \ldots, n\}$, is uniquely determined by the vectors $\bar{e}_{1}^{\prime}, \ldots, \bar{e}_{n}^{\prime}$ : it is the unique vector of $F$ which is $f$-orthogonal with every $\bar{e}_{j}^{\prime}, j \neq i$, and which satisfies $\left(\bar{e}_{i}^{\prime}, \bar{f}_{i}^{\prime \prime}\right)=1$. Hence, $\bar{f}_{i}^{\prime \prime}=\bar{f}_{i}^{\prime}$ for every $i \in\{1, \ldots, k\}$, i.e. $B_{k}=B^{\prime}$.

(2) Suppose $\left(E=E^{\prime}\right.$ and $\left.\operatorname{dim}\left(F \cap F^{\prime}\right)=n-1\right)$ or $\left(F=F^{\prime}\right.$ and $\operatorname{dim}(E \cap$ $\left.\left.E^{\prime}\right)=n-1\right)$. We will only treat the case $E=E^{\prime}$ and $\operatorname{dim}\left(F \cap F^{\prime}\right)=n-1$, since the other case is completely similar. By (1), the lemma will hold for 
the pair $\left(B, B^{\prime}\right)$ as soon as it holds for at least one pair $\left(B, B^{\prime}\right)$ giving rise to the same subspaces $E=E^{\prime}, F$ and $F^{\prime}$. So, without loss of generality, we may suppose that $B$ and $B^{\prime}$ are in such a way that $\left\{\bar{f}_{1}, \ldots, \bar{f}_{n-1}\right\}$ is a basis of $F \cap F^{\prime}$ and $\bar{e}_{i}^{\prime}=\bar{e}_{i}$ for every $i \in\{1, \ldots, n\}$. Then $\bar{f}_{i}^{\prime}=\bar{f}_{i}$ for every $i \in\{1, \ldots, n-1\}$ and there exists a $\lambda \in \mathbb{K}_{0}^{*}$ such that $\bar{f}_{n}^{\prime}=\bar{f}_{n}+\lambda \bar{e}_{n}$. So, $\left(B, B^{\prime}\right) \in \Omega_{4}$.

(3) Consider the following graph $\Gamma$. The vertices of $\Gamma$ are the pairs $(X, Y)$ where $X$ and $Y$ are two complementary totally isotropic $n$-dimensional subspaces of $V$. Two vertices $(X, Y)$ and $\left(X^{\prime}, Y^{\prime}\right)$ of $\Gamma$ are adjacent if either $\left(X=X^{\prime}\right.$ and $\left.\operatorname{dim}\left(Y \cap Y^{\prime}\right)=n-1\right)$ or $\left(Y=Y^{\prime}\right.$ and $\left.\operatorname{dim}\left(X \cap X^{\prime}\right)=n-1\right)$. We will now prove that the graph $\Gamma$ is connected. This fact, combined with (1) and (2), then finishes the proof of the lemma. Notice that the vertices of $\Gamma$ are the pairs $(x, y)$ of opposite points of $D H(2 n-1, \mathbb{K}, \psi)$. We will now prove by induction on $\mathrm{d}\left(x_{1}, x_{2}\right)$ that any two vertices $\left(x_{1}, y_{1}\right)$ and $\left(x_{2}, y_{2}\right)$ of $\Gamma$ are connected by a path.

Suppose first that $\mathrm{d}\left(x_{1}, x_{2}\right)=0$, i.e. $x_{1}=x_{2}$. Then the claim follows from the fact that the subgraph of $\Gamma$ induced on the set of points opposite to a given vertex is connected, see e.g. [6, Theorem 2.7].

Suppose $\mathrm{d}\left(x_{1}, x_{2}\right) \geq 1$. Let $x_{3}$ be a point collinear with $x_{2}$ at distance $\mathrm{d}\left(x_{1}, x_{2}\right)-1$ from $x_{1}$. By the induction hypothesis, it suffices to show that there exists a point $y_{3}$ opposite to $x_{3}$ such that $\left(x_{2}, y_{2}\right)$ and $\left(x_{3}, y_{3}\right)$ are contained in the same connected component of $\Gamma$. This clearly holds if $\mathrm{d}\left(x_{3}, y_{2}\right)=n$. (Take $\left.y_{3}=y_{2}.\right)$ Suppose therefore that $\mathrm{d}\left(x_{3}, y_{2}\right)=n-1$. Let $L$ denote a line through $y_{2}$ which is not contained in the convex subspace $\left\langle x_{3}, y_{2}\right\rangle$, and let $y_{3}$ be a point of $L \backslash\left\{y_{2}\right\}$ distinct from $\pi_{L}\left(x_{2}\right)$. Then $\mathrm{d}\left(x_{2}, y_{3}\right)=\mathrm{d}\left(x_{3}, y_{3}\right)=n$. So, $\left(x_{2}, y_{2}\right) \sim_{\Gamma}\left(x_{2}, y_{3}\right) \sim_{\Gamma}\left(x_{3}, y_{3}\right)$. This is precisely what we needed to show.

Lemma 2.2 If $\theta \in G$, then there exists an element $\eta \in \mathbb{K}^{*}$ such that $\operatorname{det}(\theta)=\frac{\eta^{\psi}}{\eta}$. The element $\eta$ is determined up to a factor of $\mathbb{K}_{0}^{*}$.

Proof. Let $B=\left(\bar{e}_{1}, \bar{f}_{1}, \ldots, \bar{e}_{n}, \bar{f}_{n}\right)$ be an arbitrary hyperbolic basis of $V$.

(i) Let $\sigma$ be a permutation of $\{1, \ldots, n\}$ and let $\theta$ be the element of $G$ mapping $B$ to $B^{\prime}=\left(\bar{e}_{\sigma(1)}, \bar{f}_{\sigma(1)}, \ldots, \bar{e}_{\sigma(n)}, \bar{f}_{\sigma(n)}\right)$. Then $\operatorname{det}(\theta)=1=\frac{1^{\psi}}{1}$.

(ii) Let $\lambda \in \mathbb{K}^{*}$ and let $\theta$ be the element of $G$ mapping $B$ to $B^{\prime}=$ $\left(\frac{\bar{e}_{1}}{\lambda}, \lambda^{\psi} \bar{f}_{1}, \bar{e}_{2}, \bar{f}_{2}, \ldots, \bar{e}_{n}, \bar{f}_{n}\right)$. Then $\operatorname{det}(\theta)=\frac{\lambda^{\psi}}{\lambda}$.

(iii) Let $\lambda \in \mathbb{K}$ and let $\theta$ be the element of $G$ mapping $B$ to $B^{\prime}=$ $\left(\bar{e}_{1}+\lambda \bar{e}_{2}, \bar{f}_{1}, \bar{e}_{2},-\lambda^{\psi} \bar{f}_{1}+\bar{f}_{2}, \bar{e}_{3}, \bar{f}_{3}, \ldots, \bar{e}_{n}, \bar{f}_{n}\right)$. Then $\operatorname{det}(\theta)=1=\frac{1^{\psi}}{1}$.

(iv) Let $\lambda \in \mathbb{K}_{0}$ and let $\theta$ be the element of $G$ mapping $B$ to $B^{\prime}=$ $\left(\bar{e}_{1}, \bar{f}_{1}, \ldots, \bar{e}_{n-1}, \bar{f}_{n-1}, \bar{e}_{n}, \bar{f}_{n}+\lambda \bar{e}_{n}\right)$. Then $\operatorname{det}(\theta)=1=\frac{1^{\psi}}{1}$. 
(v) Let $\lambda \in \mathbb{K}_{0}$ and let $\theta$ be the element of $G$ mapping $B$ to $B^{\prime}=$ $\left(\bar{e}_{1}, \bar{f}_{1}, \ldots, \bar{e}_{n-1}, \bar{f}_{n-1}, \bar{e}_{n}+\lambda \bar{f}_{n}, \bar{f}_{n}\right)$. Then $\operatorname{det}(\theta)=1=\frac{1^{\psi}}{1}$.

(vi) If $\theta_{1}, \theta_{2} \in G$ such that $\operatorname{det}\left(\theta_{i}\right)=\frac{\eta_{i}^{\psi}}{\eta_{i}}, i \in\{1,2\}$, then $\operatorname{det}\left(\theta_{2} \circ \theta_{1}\right)=$ $\operatorname{det}\left(\theta_{1}\right) \cdot \operatorname{det}\left(\theta_{2}\right)=\frac{\left(\eta_{1} \eta_{2}\right)^{\psi}}{\eta_{1} \eta_{2}}$.

The first claim of the lemma now follows from Lemma 2.1 and (i)-(vi) above. Notice also that if $\eta_{1}, \eta_{2} \in \mathbb{K}^{*}$ such that $\frac{\eta_{1}^{\psi}}{\eta_{1}}=\frac{\eta_{2}^{\psi}}{\eta_{2}}$, then $\left(\frac{\eta_{1}}{\eta_{2}}\right)^{\psi}=\frac{\eta_{1}}{\eta_{2}}$ and hence $\frac{\eta_{1}}{\eta_{2}} \in \mathbb{K}_{0}^{*}$. This also proves the second claim of the lemma.

\section{Proof of Theorem 1.1}

\subsection{A useful lemma}

Suppose that $2 \leq k \leq 2 n-1$ and that $\bar{e}_{1}$ and $\bar{f}_{1}$ are two vectors of $V$ such that $f\left(\bar{e}_{1}, \bar{f}_{1}\right)=1$. Let $V^{\prime}$ denote the set of vectors of $V$ which are $f$-orthogonal with $\bar{e}_{1}$ and $\bar{f}_{1}$ and let $f^{\prime}$ denote the skew- $\psi$-Hermitian form of $V^{\prime}$ induced by $f$. Let $G^{\prime}$ denote the group of isometries of $\left(V^{\prime}, f^{\prime}\right), H^{\prime}:=G^{\prime} \cap S L\left(V^{\prime}\right)$ and let $\widetilde{G}_{k-1}^{\prime}$ and $\widetilde{H}_{k-1}^{\prime}$ denote the subgroups of $G L\left(\bigwedge^{k-1} V^{\prime}\right)$ corresponding to $G^{\prime}$ and $H^{\prime}$ (see Section 1.1). For every vector $\alpha$ of $\bigwedge^{k-1} V^{\prime}$, let $\mu_{k}(\alpha)$ be the vector $\bar{e}_{1} \wedge \alpha$ of $\wedge^{k} V$. Then $\mu_{k}$ defines a linear isomorphism between $\bigwedge^{k-1} V^{\prime}$ and the subspace $\mu_{k}\left(\bigwedge^{k-1} V^{\prime}\right)$ of $\bigwedge^{k} V$.

Lemma 3.1 Suppose $U$ is a subspace of $\bigwedge^{k} V$ which is stabilized by $\widetilde{H}_{k}$. Then $\mu_{k}^{-1}\left(U \cap \mu_{k}\left(\bigwedge^{k-1} V^{\prime}\right)\right)$ is a subspace of $\bigwedge^{k-1} V^{\prime}$ which is stabilized by $\widetilde{H}_{k-1}^{\prime}$.

Proof. Let $\alpha$ be an arbitrary vector of $\mu_{k}^{-1}\left(U \cap \mu_{k}\left(\bigwedge^{k-1} V^{\prime}\right)\right)$ and let $\widetilde{\theta}$ be an arbitrary element of $\widetilde{H}_{k-1}^{\prime}$ corresponding to an element $\theta \in H^{\prime}$. We need to show that $\widetilde{\theta}(\alpha) \in \mu_{k}^{-1}\left(U \cap \mu_{k}\left(\bigwedge^{k-1} V^{\prime}\right)\right)$.

We extend $\theta$ to an element $\bar{\theta}$ of $H$ by defining $\bar{\theta}\left(\bar{e}_{1}\right)=\bar{e}_{1}$ and $\bar{\theta}\left(\bar{f}_{1}\right)=\bar{f}_{1}$. Let $\widetilde{\bar{\theta}}$ be the element of $\widetilde{H}_{k}$ corresponding to $\bar{\theta}$. Then for every vector $\alpha^{\prime}$ of $\bigwedge^{k-1} V^{\prime}, \mu_{k} \circ \widetilde{\theta}\left(\alpha^{\prime}\right)=\widetilde{\bar{\theta}} \circ \mu_{k}\left(\alpha^{\prime}\right)$. Hence, $\widetilde{\bar{\theta}}$ stabilizes $\mu_{k}\left(\bigwedge^{k-1} V^{\prime}\right)$.

Now, since $\mu_{k}(\alpha) \in U \cap \mu_{k}\left(\bigwedge^{k-1} V^{\prime}\right)$, also $\widetilde{\bar{\theta}} \circ \mu_{k}(\alpha) \in U \cap \mu_{k}\left(\bigwedge^{k-1} V^{\prime}\right)$. Hence, $\widetilde{\theta}(\alpha)=\mu_{k}^{-1} \circ \widetilde{\bar{\theta}} \circ \mu_{k}(\alpha) \in \mu_{k}^{-1}\left(U \cap \mu_{k}\left(\bigwedge^{k-1} V^{\prime}\right)\right)$.

\subsection{Proof of Theorem 1.1}

The following proposition is precisely Theorem 1.1 . 
Proposition 3.2 Let $k \in\{1, \ldots, 2 n\}$. If $U$ is a proper subspace of $\bigwedge^{k} V$ which is stabilized by $\widetilde{H}_{k}$, then $U=0$.

\section{Proof.}

If $k=2 n$, then $U=0$ since 0 is the only proper subspace of $\bigwedge^{2 n} V$.

Suppose $k=1$ and $U \neq 0$. Then $U$ contains a nonzero vector $\chi=\lambda_{1} \bar{e}_{1}+$ $\lambda_{1}^{\prime} \bar{f}_{1}+\cdots+\lambda_{n} \bar{e}_{n}+\lambda_{n}^{\prime} \bar{f}_{n}$, where $\left(\bar{e}_{1}, \bar{f}_{1}, \ldots, \bar{e}_{n}, \bar{f}_{n}\right)$ is some given hyperbolic basis of $V$. Without loss of generality, we may suppose that $\lambda_{1}^{\prime} \neq 0$. If $\theta$ is the element of $H$ mapping the hyperbolic basis $\left(\bar{e}_{1}, \bar{f}_{1}, \ldots, \bar{e}_{n}, \bar{f}_{n}\right)$ to the hyperbolic basis $\left(\bar{e}_{1}, \bar{e}_{1}+\bar{f}_{1}, \ldots, \bar{e}_{n}, \bar{f}_{n}\right)$, then since $\chi \in U$, also $\frac{1}{\lambda_{1}^{\prime}}(\widetilde{\theta}(\chi)-\chi)=$ $\bar{e}_{1} \in U$. Since for any $\bar{g} \in\left\{\bar{e}_{1}, \bar{f}_{1}, \ldots, \bar{e}_{n}, \bar{f}_{n}\right\}$, there exists an element of $H$ mapping $\bar{e}_{1}$ to $\bar{g}$, we have $U=\left\langle\bar{e}_{1}, \bar{f}_{1}, \ldots, \bar{e}_{n}, \bar{f}_{n}\right\rangle=V$, a contradiction.

We will now prove the lemma by induction on $n$. By the previous two paragraphs, we may suppose that $n \geq 2, k \in\{2, \ldots, 2 n-1\}$ and that the lemma holds for smaller values of $n$. Let $\left(\bar{e}_{1}, \bar{f}_{1}, \ldots, \bar{e}_{n}, \bar{f}_{n}\right)$ be a given hyperbolic basis of $V$ and let $V^{\prime}, \mu_{k}$ and $\widetilde{H}_{k-1}^{\prime}$ as in Section 3.1.

Let $\chi$ be an arbitrary vector of $U$. Then $\chi$ can be written in a unique way as

$$
\chi=\bar{e}_{1} \wedge \bar{f}_{1} \wedge \alpha(\chi)+\bar{e}_{1} \wedge \beta(\chi)+\bar{f}_{1} \wedge \gamma(\chi)+\delta(\chi),
$$

where $\alpha(\chi) \in \bigwedge^{k-2} V^{\prime}, \beta(\chi), \gamma(\chi) \in \bigwedge^{k-1} V^{\prime}$ and $\delta(\chi) \in \bigwedge^{k} V^{\prime}$. Let $\theta$ be the unique element of $H$ mapping the hyperbolic basis $\left(\bar{e}_{1}, \bar{f}_{1}, \ldots, \bar{e}_{n}, \bar{f}_{n}\right)$ of $V$ to the hyperbolic basis $\left(\bar{e}_{1}, \bar{e}_{1}+\bar{f}_{1}, \ldots, \bar{e}_{n}, \bar{f}_{n}\right)$ of $V$. Then $\widetilde{\theta}_{k}(\chi)=\chi+\bar{e}_{1} \wedge \gamma(\chi)$. Since $\chi \in U$, also $\widetilde{\theta}_{k}(\chi) \in U$ and hence also $\bar{e}_{1} \wedge \gamma(\chi) \in U$. We show that $\gamma(\chi)=0$.

Suppose $\gamma(\chi) \neq 0$. Then since $\gamma(\chi) \in \mu_{k}^{-1}\left(U \cap \mu_{k}\left(\bigwedge^{k-1} V^{\prime}\right)\right)$ and $\mu_{k}^{-1}(U \cap$ $\left.\mu_{k}\left(\bigwedge^{k-1} V^{\prime}\right)\right)$ is stabilized by $\widetilde{H}_{k-1}^{\prime}\left(\right.$ Lemma 3.1), $\mu_{k}^{-1}\left(U \cap \mu_{k}\left(\bigwedge^{k-1} V^{\prime}\right)\right)=$ $\bigwedge^{k-1} V^{\prime}$ by the induction hypothesis. So, $\mu_{k}\left(\bigwedge^{k-1} V^{\prime}\right) \subseteq U$. Hence, $U$ contains a vector of the form $\bar{e}_{1} \wedge \bar{v}_{2} \wedge \cdots \wedge \bar{v}_{k}$ where $\left\langle\bar{e}_{1}, \bar{v}_{2}, \ldots, \bar{v}_{k}\right\rangle$ is a $k$ dimensional subspace of $V$ which is totally isotropic with respect to $f$. Since $H$ acts transitively on the set of all $k$-dimensional subspaces of $V$ which are totally isotropic with respect to $f$, we would have that $U=\bigwedge^{k} V$, which is impossible.

Hence, $\gamma(\chi)=0$. In a similar way, one can prove that $\beta(\chi)=0$. What we have just done, we can also do for any pair $\left(\bar{e}_{i}, \bar{f}_{i}\right), i \in\{1, \ldots, n\}$. We can conclude:

(P1) For every $i \in\{1, \ldots, n\}$ and every $\chi \in U, \chi$ can be written in the form $\bar{e}_{i} \wedge \bar{f}_{i} \wedge \alpha_{i}(\chi)+\delta_{i}(\chi)$ where $\alpha_{i}(\chi) \in \bigwedge^{k-2}\left\langle\bar{e}_{1}, \bar{f}_{1}, \ldots, \widehat{\bar{e}}_{i}, \widehat{\bar{f}}_{i}, \ldots, \bar{e}_{n}, \bar{f}_{n}\right\rangle$ and $\delta_{i}(\chi) \in \bigwedge^{k}\left\langle\bar{e}_{1}, \bar{f}_{1}, \ldots, \widehat{\bar{e}}_{i}, \widehat{\bar{f}}_{i}, \ldots, \bar{e}_{n}, \bar{f}_{n}\right\rangle$. 
If $k$ is odd, then (P1) implies that $U=0$. Suppose therefore that $k=2 m$ is even. By (P1), every element $\chi$ of $U$ is of the form $\sum \lambda_{I} \cdot \bar{e}_{i_{1}} \wedge \bar{f}_{i_{1}} \wedge \cdots \wedge \bar{e}_{i_{m}} \wedge \bar{f}_{i_{m}}$, with the summation ranging over all subsets $I=\left\{i_{1}, \ldots, i_{m}\right\}$ of size $m$ of $\{1, \ldots, n\}$ satisfying $i_{1}<i_{2}<\cdots<i_{m}$. We will now show that all the coefficients $\lambda_{I}$ are equal to each other.

Suppose first that $I_{1}$ and $I_{2}$ are two subsets of size $m$ of $\{1,2, \ldots, n\}$ such that $\left|I_{1} \cap I_{2}\right|=m-1$. Without loss of generality, we may suppose that $I_{1} \backslash I_{2}=\{1\}$ and $I_{2} \backslash I_{1}=\{2\}$. Write $\chi=\sum \lambda_{I} \cdot \bar{e}_{i_{1}} \wedge \bar{f}_{i_{1}} \wedge \cdots \wedge \bar{e}_{i_{m}} \wedge \bar{f}_{i_{m}}$ in the form

$$
\bar{e}_{1} \wedge \bar{f}_{1} \wedge \bar{e}_{2} \wedge \bar{f}_{2} \wedge \alpha+\bar{e}_{1} \wedge \bar{f}_{1} \wedge \beta+\bar{e}_{2} \wedge \bar{f}_{2} \wedge \gamma+\delta
$$

where $\alpha \in \bigwedge^{k-4}\left\langle\bar{e}_{3}, \bar{f}_{3}, \ldots, \bar{e}_{n}, \bar{f}_{n}\right\rangle, \beta, \gamma \in \bigwedge^{k-2}\left\langle\bar{e}_{3}, \bar{f}_{3}, \ldots, \bar{e}_{n}, \bar{f}_{n}\right\rangle$ and $\delta \in$ $\bigwedge^{k}\left\langle\bar{e}_{3}, \bar{f}_{3}, \ldots, \bar{e}_{n}, \bar{f}_{n}\right\rangle$. [If $k=2$, then we omit the term $\bar{e}_{1} \wedge \bar{f}_{1} \wedge \bar{e}_{2} \wedge \bar{f}_{2} \wedge \alpha$.] Let $\theta$ denote the element of $H$ mapping the hyperbolic basis $\left(\bar{e}_{1}, \bar{f}_{1}, \bar{e}_{2}, \bar{f}_{2}, \ldots, \bar{e}_{n}\right.$, $\left.\bar{f}_{n}\right)$ of $V$ to the hyperbolic basis $\left(\bar{e}_{1}+\bar{e}_{2}, \bar{f}_{1}, \bar{e}_{2},-\bar{f}_{1}+\bar{f}_{2}, \bar{e}_{3}, \bar{f}_{3}, \ldots, \bar{e}_{n}, \bar{f}_{n}\right)$ of $V$. Then $\widetilde{\theta}(\chi)=\chi+\bar{e}_{2} \wedge \bar{f}_{1} \wedge(\beta-\gamma)$. Since $\chi \in U$, also $\widetilde{\theta}(\chi) \in U$ and hence $\bar{e}_{2} \wedge \bar{f}_{1} \wedge(\beta-\gamma) \in U$. By $(\mathrm{P} 1), \beta=\gamma$. Hence, $\lambda_{I_{1}}=\lambda_{I_{2}}$.

Consider now the most general case and let $I_{1}$ and $I_{2}$ be two arbitrary subsets of size $m$ of $\{1, \ldots, n\}$. Put $\left|I_{1} \cap I_{2}\right|=m-l$. Then there exist $l+1$ subsets $J_{0}, \ldots, J_{l}$ of size $m$ of $\{1, \ldots, n\}$ such that $J_{0}=I_{1}, J_{l}=I_{2}$ and $\left|J_{i-1} \cap J_{i}\right|=m-1$ for every $i \in\{1, \ldots, l\}$. By the previous paragraph, we know that $\lambda_{I_{1}}=\lambda_{J_{0}}=\lambda_{J_{1}}=\cdots=\lambda_{J_{l}}=\lambda_{I_{2}}$.

So, we can conclude

(P2) Every element $\chi$ of $U$ is of the form $\lambda \cdot \sum \bar{e}_{i_{1}} \wedge \bar{f}_{i_{1}} \wedge \cdots \wedge \bar{e}_{i_{m}} \wedge \bar{f}_{i_{m}}$, with the summation ranging over all subsets $I=\left\{i_{1}, \ldots, i_{m}\right\}$ of size $m$ of $\{1, \ldots, n\}$ satisfying $i_{1}<i_{2}<\cdots<i_{m}$.

Now, consider an arbitrary element $\eta \in \mathbb{K} \backslash \mathbb{K}_{0}$ satisfying $\eta^{\psi} \notin\{-\eta, \eta\}$ (if $\epsilon$ is an arbitrary element of $\mathbb{K} \backslash \mathbb{K}_{0}$, then at least one of $\epsilon, \epsilon+1$ satisfies this condition) and let $\theta^{\prime}$ be the unique element of $H$ mapping the hyperbolic basis $\left(\bar{e}_{1}, \bar{f}_{1}, \ldots, \bar{e}_{n}, \bar{f}_{n}\right)$ of $V$ to the hyperbolic basis $\left(\frac{\bar{e}_{1}}{\eta}, \eta^{\psi} \cdot \bar{f}_{1}, \eta \cdot \bar{e}_{2}, \frac{\bar{f}_{2}}{\eta^{\psi}}, \ldots, \bar{e}_{n}, \bar{f}_{n}\right)$ of $V$. Then the fact that $\widetilde{\theta}^{\prime}(\chi) \in U$ implies that the $\lambda$ mentioned in (P2) must be equal to 0 . So, $U=0$.

\section{The $\mathbb{K}_{0}$-vector space $W^{*}$}

For every hyperbolic basis $B=\left(\bar{e}_{1}, \bar{f}_{1}, \ldots, \bar{e}_{n}, \bar{f}_{n}\right)$ of $V$ and every $\lambda \in \mathbb{K} \backslash \mathbb{K}_{0}$, we will now define a basis $\mathcal{B}_{\lambda}(B)$ of $\bigwedge^{n} V$. The basis $\mathcal{B}_{\lambda}(B)$ consists of all 
the vectors

$$
\begin{gathered}
\left(\bar{g}_{\sigma(1)} \wedge \cdots \wedge \bar{g}_{\sigma(k)}\right) \wedge\left(\epsilon \cdot \bar{e}_{\sigma(k+1)} \wedge \bar{f}_{\sigma(k+1)} \wedge \cdots \wedge \bar{e}_{\sigma(k+l)} \wedge \bar{f}_{\sigma(k+l)}\right. \\
\left.+(-1)^{l} \epsilon^{\psi} \cdot \bar{e}_{\sigma(k+l+1)} \wedge \bar{f}_{\sigma(k+l+1)} \wedge \cdots \wedge \bar{e}_{\sigma(n)} \wedge \bar{f}_{\sigma(n)}\right)
\end{gathered}
$$

where $(1) k, l \in\{0, \ldots, n\}$ such that $k+2 l=n,(2) \in \in\{1, \lambda\},(3) \bar{g}_{i} \in\left\{\bar{e}_{i}, \bar{f}_{i}\right\}$ for every $i \in\{\sigma(1), \ldots, \sigma(k)\}$, (4) $\sigma$ is a permutation of $\{1, \ldots, n\}$ satisfying (i) $\sigma(1)<\sigma(2)<\cdots<\sigma(k)$, (ii) $\sigma(k+1)<\sigma(k+2)<\cdots<\sigma(k+l)$, (iii) $\sigma(k+l+1)<\sigma(k+l+2)<\cdots<\sigma(n)$, (iv) $\sigma(k+1)<\sigma(k+l+1)$.

Let $W_{\lambda}(B)$ denote the set of all $\mathbb{K}_{0}$-linear combinations of the elements of $\mathcal{B}_{\lambda}(B)$. Now, for all $\lambda_{1}, \lambda_{2} \in \mathbb{K} \backslash \mathbb{K}_{0}$, there exist $a_{1}, a_{2} \in \mathbb{K}_{0}$ such that $\left(\lambda_{2}, \lambda_{2}^{\psi}\right)=a_{1}(1,1)+a_{2}\left(\lambda_{1}, \lambda_{1}^{\psi}\right)$. From this we readily observe that $W_{\lambda_{1}}(B)=$ $W_{\lambda_{2}}(B)$ for any two $\lambda_{1}, \lambda_{2} \in \mathbb{K} \backslash \mathbb{K}_{0}$. We define $W(B):=W_{\lambda}(B)$ where $\lambda$ is an arbitrary element of $\mathbb{K} \backslash \mathbb{K}_{0}$.

Lemma 4.1 If $B_{1}$ and $B_{2}$ are two hyperbolic bases of $V$ such that $\left(B_{1}, B_{2}\right) \in$ $\Omega_{1} \cup \Omega_{2} \cup \Omega_{3} \cup \Omega_{4} \cup \Omega_{5}$ and if $\theta$ is the unique element of $G$ mapping $B_{1}$ to $B_{2}$, then $W\left(B_{2}\right)=\left\{\frac{\alpha}{\eta_{\theta}} \mid \alpha \in W\left(B_{1}\right)\right\}$. In particular, if $\left(B_{1}, B_{2}\right) \in \Omega_{1} \cup \Omega_{3} \cup$ $\Omega_{4} \cup \Omega_{5}$, then $W\left(B_{2}\right)=W\left(B_{1}\right)$.

Proof. Put $B_{1}=\left(\bar{e}_{1}, \bar{f}_{1}, \ldots, \bar{e}_{n}, \bar{f}_{n}\right)$ and $B_{2}=\left(\bar{e}_{1}^{\prime}, \bar{f}_{1}^{\prime}, \ldots, \bar{e}_{n}^{\prime}, \bar{f}_{n}^{\prime}\right)$. Notice that $\eta_{\theta} \cdot \eta_{\theta^{-1}} \in \mathbb{K}_{0}^{*}$ and if $\left(B_{1}, B_{2}\right) \in \Omega_{i}, i \in\{1, \ldots, 5\}$, then also $\left(B_{2}, B_{1}\right) \in \Omega_{i}$. So, it suffices to prove that $W\left(B_{2}\right) \subseteq\left\{\frac{\alpha}{\eta_{\theta}} \mid \alpha \in W\left(B_{1}\right)\right\}$, or equivalently, that $\mathcal{B}_{\lambda}\left(B_{2}\right) \subseteq\left\{\frac{\alpha}{\eta_{\theta}} \mid \alpha \in W\left(B_{1}\right)\right\}$, where $\lambda$ is a given element of $\mathbb{K} \backslash \mathbb{K}_{0}$. The latter statement is easily seen to be true if $\left(B_{1}, B_{2}\right) \in \Omega_{1} \cup \Omega_{2} \cup \Omega_{4} \cup \Omega_{5}$. We will now treat the harder case $\left(B_{1}, B_{2}\right) \in \Omega_{3}$. Then there exists a $\eta \in \mathbb{K}$ such that $\bar{e}_{1}^{\prime}=\bar{e}_{1}+\eta \bar{e}_{2}, \bar{f}_{1}^{\prime}=\bar{f}_{1}, \bar{e}_{2}^{\prime}=\bar{e}_{2}, \bar{f}_{2}^{\prime}=-\eta^{\psi} \bar{f}_{1}+\bar{f}_{2}, \bar{e}_{3}^{\prime}=\bar{e}_{3}, \bar{f}_{3}^{\prime}=\bar{f}_{3}, \ldots$, $\bar{e}_{n}^{\prime}=\bar{e}_{n}$ and $\bar{f}_{n}^{\prime}=\bar{f}_{n}$. Let

$$
\begin{gathered}
\chi=\left(\bar{g}_{\sigma(1)}^{\prime} \wedge \cdots \wedge \bar{g}_{\sigma(k)}^{\prime}\right) \wedge\left(\epsilon \cdot \bar{e}_{\sigma(k+1)}^{\prime} \wedge \bar{f}_{\sigma(k+1)}^{\prime} \wedge \cdots \wedge \bar{e}_{\sigma(k+l)}^{\prime} \wedge \bar{f}_{\sigma(k+l)}^{\prime}\right. \\
\left.+(-1)^{l} \epsilon^{\psi} \cdot \bar{e}_{\sigma(k+l+1)}^{\prime} \wedge \bar{f}_{\sigma(k+l+1)}^{\prime} \wedge \cdots \wedge \bar{e}_{\sigma(n)}^{\prime} \wedge \bar{f}_{\sigma(n)}^{\prime}\right)
\end{gathered}
$$

be an arbitrary element of $\mathcal{B}_{\lambda}\left(B_{2}\right)$, where $(1) k, l \in\{0, \ldots, n\}$ such that $k+2 l=n,(2) \epsilon \in\{1, \lambda\},(3) \bar{g}_{i}^{\prime} \in\left\{\bar{e}_{i}^{\prime}, \bar{f}_{i}^{\prime}\right\}$ for every $i \in\{\sigma(1), \ldots, \sigma(k)\},(4)$ $\sigma$ is a permutation of $\{1, \ldots, n\}$ satisfying (i) $\sigma(1)<\sigma(2)<\cdots<\sigma(k)$, (ii) $\sigma(k+1)<\sigma(k+2)<\cdots<\sigma(k+l)$, (iii) $\sigma(k+l+1)<\sigma(k+l+2)<\cdots<\sigma(n)$, (iv) $\sigma(k+1)<\sigma(k+l+1)$. There are 10 possibilities:

(1) Suppose $\sigma(1)=1, \sigma(2)=2, \bar{g}_{1}^{\prime}=\bar{e}_{1}^{\prime}$ and $\bar{g}_{2}^{\prime}=\bar{e}_{2}^{\prime}$. Then $\chi$ can be written in a natural way as $\chi=\bar{e}_{1}^{\prime} \wedge \bar{e}_{2}^{\prime} \wedge \alpha \wedge \beta$, where $\alpha=\bar{g}_{\sigma(3)}^{\prime} \wedge \cdots \wedge \bar{g}_{\sigma(k)}^{\prime}$ 
and $\beta$ does not involve indices which are equal to either 1 or 2 . We have $\chi=\left(\bar{e}_{1}+\eta \bar{e}_{2}\right) \wedge \bar{e}_{2} \wedge \alpha \wedge \beta=\bar{e}_{1} \wedge \bar{e}_{2} \wedge \alpha \wedge \beta \in \mathcal{B}_{\lambda}\left(B_{1}\right)$.

(2) Suppose $\sigma(1)=1, \sigma(2)=2, \bar{g}_{1}^{\prime}=\bar{e}_{1}^{\prime}$ and $\bar{g}_{2}^{\prime}=\bar{f}_{2}^{\prime}$. Then $\chi$ can be written in a natural way as $\chi=\bar{e}_{1}^{\prime} \wedge \bar{f}_{2}^{\prime} \wedge \alpha \wedge\left(\epsilon \cdot \beta+(-1)^{l} \epsilon^{\psi} \cdot \gamma\right)$, where $\alpha, \beta$ and $\gamma$ do not involve indices which are equal to 1 or 2 . We have $\chi=\left(\bar{e}_{1}+\eta \bar{e}_{2}\right) \wedge\left(-\eta^{\psi} \bar{f}_{1}+\bar{f}_{2}\right) \wedge \alpha \wedge\left(\epsilon \cdot \beta+(-1)^{l} \epsilon^{\psi} \cdot \gamma\right)=\bar{e}_{1} \wedge \bar{f}_{2} \wedge \alpha \wedge(\epsilon \cdot \beta+$ $\left.(-1)^{l} \epsilon^{\psi} \cdot \gamma\right)-\eta^{\psi+1} \bar{e}_{2} \wedge \bar{f}_{1} \wedge \alpha \wedge\left(\epsilon \cdot \beta+(-1)^{l} \epsilon^{\psi} \cdot \gamma\right)+\alpha \wedge\left(-\eta^{\psi} \epsilon \cdot \bar{e}_{1} \wedge \bar{f}_{1} \wedge \beta-\right.$ $\left.(-1)^{l+1}\left(\eta^{\psi} \epsilon\right)^{\psi} \cdot \bar{e}_{2} \wedge \bar{f}_{2} \wedge \gamma\right)+\alpha \wedge\left(\epsilon \eta \cdot \bar{e}_{2} \wedge \bar{f}_{2} \wedge \beta+(-1)^{l+1}(\epsilon \eta)^{\psi} \cdot \bar{e}_{1} \wedge \bar{f}_{1} \wedge \gamma\right)$ and this is clearly a $\mathbb{K}_{0}$-linear combination of elements of $\mathcal{B}_{\lambda}\left(B_{1}\right)$.

(3) Suppose $\sigma(1)=1, \sigma(2)=2, \bar{g}_{1}^{\prime}=\bar{f}_{1}^{\prime}$ and $\bar{g}_{2}^{\prime}=\bar{e}_{2}^{\prime}$. Then $\chi$ can be written in a natural way as $\chi=\bar{f}_{1}^{\prime} \wedge \bar{e}_{2}^{\prime} \wedge \alpha \wedge \beta$, where $\alpha=\bar{g}_{\sigma(3)}^{\prime} \wedge \cdots \wedge \bar{g}_{\sigma(k)}^{\prime}$ and $\beta$ does not involve indices which are equal to either 1 or 2 . We have $\chi=\bar{f}_{1} \wedge \bar{e}_{2} \wedge \alpha \wedge \beta \in \mathcal{B}_{\lambda}\left(B_{1}\right)$.

(4) Suppose $\sigma(1)=1, \sigma(2)=2, \bar{g}_{1}^{\prime}=\bar{f}_{1}^{\prime}$ and $\bar{g}_{2}^{\prime}=\bar{f}_{2}^{\prime}$. Then $\chi$ can be written in a natural way as $\chi=\bar{f}_{1}^{\prime} \wedge \bar{f}_{2}^{\prime} \wedge \alpha \wedge \beta$, where $\alpha=\bar{g}_{\sigma(3)}^{\prime} \wedge \cdots \wedge \bar{g}_{\sigma(k)}^{\prime}$ and $\beta$ does not involve indices which are equal to either 1 or 2 . We have $\chi=\bar{f}_{1} \wedge\left(-\eta^{\psi} \bar{f}_{1}+\bar{f}_{2}\right) \wedge \alpha \wedge \beta=\bar{f}_{1} \wedge \bar{f}_{2} \wedge \alpha \wedge \beta \in \mathcal{B}_{\lambda}\left(B_{1}\right)$.

(5) Suppose $\sigma(1)=1, \sigma(k+1)=2$ and $\bar{g}_{1}^{\prime}=\bar{e}_{1}^{\prime}$. Then $\chi$ can be written in a natural way as $\chi=\bar{e}_{1}^{\prime} \wedge \alpha \wedge\left(\epsilon \cdot \bar{e}_{2}^{\prime} \wedge \bar{f}_{2}^{\prime} \wedge \beta+(-1)^{l} \epsilon^{\psi} \cdot \gamma\right)$ where $\alpha, \beta$ and $\gamma$ does not involve indices which are equal to 1 or 2 . We have $\chi=\left(\bar{e}_{1}+\eta \bar{e}_{2}\right) \wedge \alpha \wedge\left(\epsilon \cdot \bar{e}_{2} \wedge\left(-\eta^{\psi} \bar{f}_{1}+\bar{f}_{2}\right) \wedge \beta+(-1)^{l} \epsilon^{\psi} \cdot \gamma\right)=\bar{e}_{1} \wedge \alpha \wedge(\epsilon$. $\left.\bar{e}_{2} \wedge \bar{f}_{2} \wedge \beta+(-1)^{l} \epsilon^{\psi} \cdot \gamma\right)+\bar{e}_{2} \wedge \alpha \wedge\left(\epsilon \eta^{\psi} \cdot \bar{e}_{1} \wedge \bar{f}_{1} \wedge \beta+(-1)^{l}\left(\epsilon \eta^{\psi}\right)^{\psi} \cdot \gamma\right)$ and this is clearly a $\mathbb{K}_{0}$-linear combination of the elements of $\mathcal{B}_{\lambda}\left(B_{1}\right)$.

(6) Suppose $\sigma(1)=1, \sigma(k+1)=2$ and $\bar{g}_{1}^{\prime}=\bar{f}_{1}^{\prime}$. Then $\chi$ can be written in a natural way as $\chi=\bar{f}_{1}^{\prime} \wedge \alpha \wedge\left(\epsilon \cdot \bar{e}_{2}^{\prime} \wedge \bar{f}_{2}^{\prime} \wedge \beta+(-1)^{l} \epsilon^{\psi} \cdot \gamma\right)$ where $\alpha, \beta$ and $\gamma$ does not involve induces which are equal to 1 or 2 . We have $\chi=\bar{f}_{1} \wedge \alpha \wedge\left(\epsilon \cdot \bar{e}_{2} \wedge\right.$ $\left.\left(-\eta^{\psi} \bar{f}_{1}+\bar{f}_{2}\right) \wedge \beta+(-1)^{l} \epsilon^{\psi} \cdot \gamma\right)=\bar{f}_{1} \wedge \alpha \wedge\left(\epsilon \cdot \bar{e}_{2} \wedge \bar{f}_{2} \wedge \beta+(-1)^{l} \epsilon^{\psi} \cdot \gamma\right) \in \mathcal{B}_{\lambda}\left(B_{1}\right)$.

(7) Suppose $\sigma(1)=2, \sigma(k+1)=1$ and $\bar{g}_{2}^{\prime}=\bar{e}_{2}^{\prime}$. Then $\chi$ can be written in natural way as $\chi=\bar{e}_{2}^{\prime} \wedge \alpha \wedge\left(\epsilon \cdot \bar{e}_{1}^{\prime} \wedge \bar{f}_{1}^{\prime} \wedge \beta+(-1)^{l} \epsilon^{\psi} \cdot \gamma\right)$, where $\alpha, \beta$ and $\gamma$ do not involve indices which are equal to 1 or 2 . We have $\chi=\bar{e}_{2} \wedge \alpha \wedge\left(\epsilon \cdot\left(\bar{e}_{1}+\right.\right.$ $\left.\left.\eta \bar{e}_{2}\right) \wedge \bar{f}_{1} \wedge \beta+(-1)^{l} \epsilon^{\psi} \cdot \gamma\right)=\bar{e}_{2} \wedge \alpha \wedge\left(\epsilon \cdot \bar{e}_{1} \wedge \bar{f}_{1} \wedge \beta+(-1)^{l} \epsilon^{\psi} \cdot \gamma\right) \in \mathcal{B}_{\lambda}\left(B_{1}\right)$.

(8) Suppose $\sigma(1)=2, \sigma(k+1)=1$ and $\bar{g}_{2}^{\prime}=\bar{f}_{2}^{\prime}$. Then $\chi$ can be written in a natural way as $\bar{f}_{2}^{\prime} \wedge \alpha \wedge\left(\epsilon \cdot \bar{e}_{1}^{\prime} \wedge \bar{f}_{1}^{\prime} \wedge \beta+(-1)^{l} \epsilon^{\psi} \cdot \gamma\right)$, where $\alpha, \beta$ and $\gamma$ do not involve indices which are equal to 1 of 2 . We have $\chi=\left(-\eta^{\psi} \bar{f}_{1}+\bar{f}_{2}\right) \wedge \alpha \wedge\left(\epsilon \cdot\left(\bar{e}_{1}+\eta \bar{e}_{2}\right) \wedge \bar{f}_{1} \wedge \beta+(-1)^{l} \epsilon^{\psi} \cdot \gamma\right)=\bar{f}_{2} \wedge \alpha \wedge$ $\left(\epsilon \cdot \bar{e}_{1} \wedge \bar{f}_{1} \wedge \beta+(-1)^{l} \epsilon^{\psi} \cdot \gamma\right)-\bar{f}_{1} \wedge \alpha \wedge\left(\epsilon \eta \cdot \bar{e}_{2} \wedge \bar{f}_{2} \wedge \beta+(-1)^{l}(\epsilon \eta)^{\psi} \cdot \gamma\right)$ and this is clearly a $\mathbb{K}_{0}$-linear combination of elements of $\mathcal{B}_{\lambda}\left(B_{1}\right)$.

(9) Suppose $\sigma(k+1)=1$ and $\sigma(k+2)=2$. Then $\chi$ can be written in a natural way as $\chi=\alpha \wedge\left(\epsilon \cdot \bar{e}_{1}^{\prime} \wedge \bar{f}_{1}^{\prime} \wedge \bar{e}_{2}^{\prime} \wedge \bar{f}_{2}^{\prime} \wedge \beta+(-1)^{l} \epsilon^{\psi} \cdot \gamma\right)$ where $\alpha, \beta$ and $\gamma$ do not involve indices which are equal to 1 or 2 . We have $\chi=\alpha \wedge\left(\epsilon \cdot\left(\bar{e}_{1}+\eta \bar{e}_{2}\right) \wedge \bar{f}_{1} \wedge \bar{e}_{2} \wedge\left(-\eta^{\psi} \bar{f}_{1}+f_{2}\right) \wedge \beta+(-1)^{l} \epsilon^{\psi} \cdot \gamma\right)=\alpha \wedge(\epsilon$. 
$\left.\bar{e}_{1} \wedge \bar{f}_{1} \wedge \bar{e}_{2} \wedge \bar{f}_{2} \wedge \beta+(-1)^{l} \epsilon^{\psi} \cdot \gamma\right) \in \mathcal{B}_{\lambda}\left(B_{1}\right)$.

(10) Suppose $\sigma(k+1)=1$ and $\sigma(k+l+1)=2$. Then $\chi$ can be written in a natural way as $\chi=\alpha \wedge\left(\epsilon \cdot \bar{e}_{1} \wedge \bar{f}_{1} \wedge \beta+(-1)^{l} \epsilon^{\psi} \cdot \bar{e}_{2} \wedge \bar{f}_{2} \wedge \gamma\right)$, where $\alpha, \beta$ and $\gamma$ do not involve indices which are equal to 1 or 2 . We have $\chi=\alpha \wedge\left(\epsilon \cdot\left(\bar{e}_{1}+\eta \bar{e}_{2}\right) \wedge \bar{f}_{1} \wedge \beta+(-1)^{l} \epsilon^{\psi} \cdot \bar{e}_{2} \wedge\left(-\eta^{\psi} \bar{f}_{1}+\bar{f}_{2}\right) \wedge \gamma\right)=\alpha \wedge(\epsilon$. $\left.\bar{e}_{1} \wedge \bar{f}_{1} \wedge \beta+(-1)^{l} \epsilon^{\psi} \cdot \bar{e}_{2} \wedge \bar{f}_{2} \wedge \gamma\right)-\bar{f}_{1} \wedge \bar{e}_{2} \wedge \alpha \wedge\left(\epsilon \eta \cdot \beta+(-1)^{l+1}(\epsilon \eta)^{\psi} \cdot \gamma\right)$ and this is clearly a $\mathbb{K}_{0}$-linear combination of elements of $\mathcal{B}_{\lambda}\left(B_{1}\right)$.

Lemma 4.2 If $B_{1}$ and $B_{2}$ are two hyperbolic bases of $V$ and if $\theta$ is the unique element of $G$ mapping $B_{1}$ to $B_{2}$, then $W\left(B_{2}\right)=\left\{\frac{\alpha}{\eta_{\theta}} \mid \alpha \in W\left(B_{1}\right)\right\}$.

Proof. Let $B_{1}, B_{2}$ and $B_{3}$ be three hyperbolic bases of $V$ and let $\theta_{i}, i \in$ $\{1,2\}$, be the unique element of $G$ mapping $B_{i}$ to $B_{i+1}$. Then $\theta_{3}:=\theta_{2} \circ \theta_{1}$ is the unique element of $G$ mapping $B_{1}$ to $B_{3}$. In view of Lemmas 2.1 and 4.1 , it suffices to show that if the lemma holds for the pairs $\left(B_{1}, B_{2}\right)$ and $\left(B_{2}, B_{3}\right)$, then it also holds for the pair $\left(B_{1}, B_{3}\right)$. As remarked in Section 1.1, $\eta_{\theta_{3}} \cdot \eta_{\theta_{2}}^{-1} \cdot \eta_{\theta_{1}}^{-1} \in \mathbb{K}_{0}$. Now, since $W_{B_{3}}=\left\{\frac{\alpha}{\eta_{\theta_{2}}} \mid \alpha \in W\left(B_{2}\right)\right\}$ and $W_{B_{2}}=\left\{\frac{\alpha}{\eta_{\theta_{1}}} \mid \alpha \in\right.$ $\left.W\left(B_{1}\right)\right\}$, we have $W_{B_{3}}=\left\{\frac{\alpha}{\eta_{\theta_{1}} \cdot \eta_{\theta_{2}}} \mid \alpha \in W\left(B_{1}\right)\right\}=\left\{\frac{\alpha}{\eta_{\theta_{3}}} \mid \alpha \in W\left(B_{1}\right)\right\}$.

Now, let $B^{*}$ be a fixed hyperbolic basis of $V$ and put $W^{*}:=W\left(B^{*}\right)$. Then Theorem 1.3 is an immediate consequence of Lemma 4.2. (Notice that since $\widetilde{\theta}_{n}$ maps every element of $\mathcal{B}_{\lambda}\left(B^{*}\right)$ to an element of $\mathcal{B}_{\lambda}\left(\theta\left(B^{*}\right)\right)$, we have $\widetilde{\theta}_{n}\left(W^{*}\right)=W\left(\theta\left(B^{*}\right)\right)$.) We will now also give a proof of Theorem 1.6.

Proof. Let $\left\langle\bar{e}_{1}, \bar{e}_{2}, \ldots, \bar{e}_{n}\right\rangle$ be an $n$-dimensional subspace of $V$ which is totally isotropic with respect to $f$. Extend $\left(\bar{e}_{1}, \bar{e}_{2}, \ldots, \bar{e}_{n}\right)$ to a hyperbolic basis $B=\left(\bar{e}_{1}, \bar{f}_{1}, \ldots, \bar{e}_{n}, \bar{f}_{n}\right)$ of $V$. Then $\bar{e}_{1} \wedge \bar{e}_{2} \wedge \cdots \wedge \bar{e}_{n} \in W(B)$. Claim (1) of Theorem 1.6 now follows from Lemma 4.2.

Let $\left\langle\bar{e}_{1}, \bar{e}_{2}, \ldots, \bar{e}_{n-1}\right\rangle$ be an $(n-1)$-dimensional subspace of $V$ which is totally isotropic with respect to $f$. Let $\bar{e}_{n}$ and $\bar{f}_{n}$ be two vectors of $V$ which are $f$-orthogonal with $\left\langle\bar{e}_{1}, \ldots, \bar{e}_{n-1}\right\rangle$ and which satisfy $f\left(\bar{e}_{n}, \bar{f}_{n}\right)=1$. Then the $n$-dimensional subspaces of $V$ through $\left\langle\bar{e}_{1}, \bar{e}_{2}, \ldots, \bar{e}_{n-1}\right\rangle$ which are totally isotropic with respect to $f$ are precisely the subspaces $\left\langle\bar{e}_{1}, \ldots, \bar{e}_{n}\right\rangle$, $\left\langle\bar{e}_{1}, \ldots, \bar{e}_{n-1}, \bar{f}_{n}+\lambda \bar{e}_{n}\right\rangle, \lambda \in \mathbb{K}_{0}$. Now, extend $\left(\bar{e}_{1}, \ldots, \bar{e}_{n}, \bar{f}_{n}\right)$ to a hyperbolic basis $B=\left(\bar{e}_{1}, \bar{f}_{1}, \ldots, \bar{e}_{n}, \bar{f}_{n}\right)$ of $V$. Then for every $\lambda \in \mathbb{K}_{0}$, also $B_{\lambda}:=\left(\bar{e}_{1}, \bar{f}_{1}, \ldots, \bar{e}_{n-1}, \bar{f}_{n-1}, \bar{e}_{n}, \bar{f}_{n}+\lambda \bar{e}_{n}\right)$ is a hyperbolic basis of $V$. Now, by Lemma $4.1, W\left(B_{\lambda}\right)=W(B)$ for every $\lambda \in \mathbb{K}_{0}$. Now, $\bar{e}_{1} \wedge \bar{e}_{2} \wedge \cdots \wedge \bar{e}_{n} \in W(B)$ and $\bar{e}_{1} \wedge \cdots \wedge \bar{e}_{n-1} \wedge\left(\bar{f}_{n}+\lambda \bar{e}_{n}\right)=\bar{e}_{1} \wedge \cdots \wedge \bar{e}_{n-1} \wedge \bar{f}_{n}+\lambda \cdot \bar{e}_{1} \wedge \bar{e}_{2} \wedge \cdots \wedge \bar{e}_{n} \in$ $W\left(B_{\lambda}\right)=W(B)$. It now follows that the line of $D H(2 n-1, \mathbb{K}, \psi)$ corresponding to the subspace $\left\langle\bar{e}_{1}, \bar{e}_{2}, \ldots, \bar{e}_{n-1}\right\rangle$ is mapped by $e$ to a line of $\operatorname{PG}\left(W^{*}\right)$. 
Now, let $B=\left(\bar{e}_{1}, \bar{f}_{1}, \ldots, \bar{e}_{n}, \bar{f}_{n}\right)$ be a hyperbolic basis of $V$. For every two vectors $\alpha_{1}$ and $\alpha_{2}$ of $\bigwedge^{n} V$, we define $\widetilde{f}_{B}\left(\alpha_{1}, \alpha_{2}\right) \in \mathbb{K}$ in such a way that

$$
\alpha_{1} \wedge \alpha_{2}=\tilde{f}_{B}\left(\alpha_{1}, \alpha_{2}\right) \cdot\left(\bar{e}_{1} \wedge \bar{f}_{1}\right) \wedge \cdots \wedge\left(\bar{e}_{n} \wedge \bar{f}_{n}\right) .
$$

Clearly, $\widetilde{f}_{B}$ is a nondegenerate form which is symmetric if $n$ is even and alternating if $n$ is odd.

Lemma 4.3 (1) If $\theta \in G$ and $B$ is a hyperbolic basis, then $\widetilde{f}_{B}=\operatorname{det}(\theta) \cdot \widetilde{f}_{\theta(B)}$. In particular, if $\theta \in H$, then $\widetilde{f}_{B}=\widetilde{f}_{\theta(B)}$.

(2) If $B$ is a hyperbolic basis and $\alpha_{1}, \alpha_{2} \in W(B)$, then $\tilde{f}_{B}\left(\alpha_{1}, \alpha_{2}\right) \in \mathbb{K}_{0}$.

Proof. (1) If $\alpha_{1}, \alpha_{2} \in \wedge^{n} V$, then $\tilde{f}_{B}\left(\alpha_{1}, \alpha_{2}\right) \cdot\left(\bar{e}_{1} \wedge \bar{f}_{1}\right) \wedge \cdots \wedge\left(\bar{e}_{n} \wedge \bar{f}_{n}\right)=$ $\alpha_{1} \wedge \alpha_{2}=\widetilde{f}_{\theta(B)}\left(\alpha_{1}, \alpha_{2}\right) \cdot \theta\left(\bar{e}_{1}\right) \wedge \theta\left(\bar{f}_{1}\right) \wedge \cdots \wedge \theta\left(\bar{e}_{n}\right) \wedge \theta\left(\bar{f}_{n}\right)=\operatorname{det}(\theta) \cdot \widetilde{f}_{\theta(B)}\left(\alpha_{1}, \alpha_{2}\right)$. $\bar{e}_{1} \wedge \bar{f}_{1} \wedge \cdots \wedge \bar{e}_{n} \wedge \bar{f}_{n}$. Hence, $\widetilde{f}_{B}\left(\alpha_{1}, \alpha_{2}\right)=\operatorname{det}(\theta) \cdot \widetilde{f}_{\theta(B)}\left(\alpha_{1}, \alpha_{2}\right)$.

(2) Let $\lambda$ be an arbitrary element of $\mathbb{K} \backslash \mathbb{K}_{0}$. It suffices to prove that $\widetilde{f}\left(\alpha_{1}, \alpha_{2}\right) \in \mathbb{K}_{0}$ for every two vectors $\alpha_{1}, \alpha_{2} \in \mathcal{B}_{\lambda}(B)$. We readily observe that $\widetilde{f}\left(\alpha_{1}, \alpha_{2}\right)$ is always equal to 0 if $\alpha_{1}, \alpha_{2} \in \mathcal{B}_{\lambda}(B)$, except in the following cases:

(a) $\alpha_{1}=\bar{g}_{1} \wedge \bar{g}_{2} \wedge \cdots \wedge \bar{g}_{n}$ and $\alpha_{2}=\bar{g}_{1}^{\prime} \wedge \bar{g}_{2}^{\prime} \wedge \cdots \wedge \bar{g}_{n}^{\prime}$ where $\left\{\bar{g}_{i}, \bar{g}_{i}^{\prime}\right\}=\left\{\bar{e}_{i}, \bar{f}_{i}\right\}$ for every $i \in\{1, \ldots, n\}$. One readily verifies that $\widetilde{f}_{B}\left(\alpha_{1}, \alpha_{2}\right) \in\{-1,1\} \subseteq \mathbb{K}_{0}$.

(b) $\alpha_{1}=\left(\bar{g}_{\sigma(1)} \wedge \cdots \wedge \bar{g}_{\sigma(k)}\right) \wedge\left(\epsilon \cdot \bar{e}_{\sigma(k+1)} \wedge \bar{f}_{\sigma(k+1)} \wedge \cdots \wedge \bar{e}_{\sigma(k+l)} \wedge \bar{f}_{\sigma(k+l)}+\right.$ $\left.(-1)^{l} \epsilon^{\psi} \cdot \bar{e}_{\sigma(k+l+1)} \wedge \bar{f}_{\sigma(k+l+1)} \wedge \cdots \wedge \bar{e}_{\sigma(n)} \wedge \bar{f}_{\sigma(n)}\right)$ and $\alpha_{2}=\left(\bar{g}_{\sigma(1)}^{\prime} \wedge \cdots \wedge \bar{g}_{\sigma(k)}^{\prime}\right) \wedge$ $\left(\epsilon^{\prime} \cdot \bar{e}_{\sigma(k+1)} \wedge \bar{f}_{\sigma(k+1)} \wedge \cdots \wedge \bar{e}_{\sigma(k+l)} \wedge \bar{f}_{\sigma(k+l)}+(-1)^{l} \epsilon^{\prime \psi} \cdot \bar{e}_{\sigma(k+l+1)} \wedge \bar{f}_{\sigma(k+l+1)} \wedge\right.$ $\left.\cdots \wedge \bar{e}_{\sigma(n)} \wedge \bar{f}_{\sigma(n)}\right)$, where $(1) k, l \in\{0, \ldots, n\}$ such that $l>0$ and $k+2 l=n$, (2) $\epsilon, \epsilon^{\prime} \in\{1, \lambda\},(3)\left\{\bar{g}_{i}, \bar{g}_{i}^{\prime}\right\}=\left\{\bar{e}_{i}, \bar{f}_{i}\right\}$ for every $i \in\{\sigma(1), \ldots, \sigma(k)\}$, (4) $\sigma$ is a permutation of $\{1, \ldots, n\}$ satisfying (i) $\sigma(1)<\sigma(2)<\cdots<\sigma(k)$, (ii) $\sigma(k+1)<\sigma(k+2)<\cdots<\sigma(k+l)$, (iii) $\sigma(k+l+1)<\sigma(k+l+$ 2) $<\cdots<\sigma(n)$, (iv) $\sigma(k+1)<\sigma(k+l+1)$. One readily verifies that $\widetilde{f}_{B}\left(\alpha_{1}, \alpha_{2}\right) \in\left\{\left(\epsilon \cdot \epsilon^{\prime \psi}+\epsilon^{\psi} \epsilon^{\prime}\right),-\left(\epsilon \cdot \epsilon^{\prime \psi}+\epsilon^{\psi} \epsilon^{\prime}\right)\right\} \subseteq \mathbb{K}_{0}$.

Again consider a fixed hyperbolic basis $B^{*}$ of $V$ and let $\tilde{f}^{*}$ be the restriction of $\widetilde{f}_{B^{*}}$ to the $\mathbb{K}_{0}$-vector space $W^{*}=W\left(B^{*}\right)$. Then $\widetilde{f}^{*}$ is a nondegenerate bilinear form on the vector space $W^{*}$. This form defines a polarity $\zeta^{*}$ of $\operatorname{PG}\left(W^{*}\right)$. If $n$ is odd or $\operatorname{char}(\mathbb{K})=2$, then $\zeta^{*}$ is a symplectic polarity. Otherwise, $\zeta^{*}$ is an orthogonal polarity. If $U$ is a subspace of $W^{*}$, then we define $U^{\perp}:=\left\{x \in W^{*} \mid \tilde{f}^{*}(x, u)=0, \forall u \in U\right\}$. 
Remark. If $e: \Delta \rightarrow \Sigma$ is the so-called minimal full polarized embedding (see [4] for the definition) of a thick dual polar space $\Delta$ in a finite-dimensional projective space $\Sigma$, then there exists a unique polarity $\zeta$ of $\Sigma$ such that two points $p_{1}$ and $p_{2}$ of $\Delta$ are not opposite if and only if $e\left(p_{2}\right) \in e\left(p_{1}\right)^{\zeta}$. The polarity $\zeta^{*}$ defined above is a special case of this (take $e=e_{g r}, \Delta=$ $D H(2 n-1, \mathbb{K}, \psi)$ and $\left.\Sigma=\operatorname{PG}\left(W^{*}\right)\right)$. We refer to Cardinali, De Bruyn and Pasini [4] for more information on minimal full polarized embeddings. The existence of the polarity $\zeta$ is an immediate consequence of the isomorphism between the embedding $e$ and its so-called dual embedding $e^{*}$.

\section{$5 \quad$ Hyperplanes of $D H(2 n-1, \mathbb{K}, \psi)$}

\subsection{Representative vectors}

By Shult [11, Lemma 6.1], every hyperplane of a thick dual polar space (in particular, of $D H(2 n-1, \mathbb{K}, \psi)$ ) is a maximal subspace. So, if $H$ is a hyperplane of $D H(2 n-1, \mathbb{K}, \psi)$ arising from $e_{g r}$, then $\left\langle e_{g r}(H)\right\rangle$ necessarily is a hyperplane of $\mathrm{PG}\left(W^{*}\right)$ and there exists a unique 1-space $U$ of $W^{*}$ such that $\left\langle e_{g r}(H)\right\rangle=\mathrm{PG}\left(U^{\perp}\right)$. Any nonzero vector of $U$ is called a representative vector of $H$.

Consider now the special case $n=3$. Recall that $B^{*}=\left(\bar{e}_{1}, \bar{f}_{1}, \bar{e}_{2}, \bar{f}_{2}, \bar{e}_{3}, \bar{f}_{3}\right)$ is a given hyperbolic basis of $V$ and that $W^{*}=W\left(B^{*}\right)$. Let $\lambda$ be an arbitrary point of $\mathbb{K} \backslash \mathbb{K}_{0}$. By Section 4 , a basis of the $\mathbb{K}_{0}$-vector space $W^{*}$ is given by the following 20 vectors:

$$
\begin{gathered}
\bar{e}_{1} \wedge \bar{e}_{2} \wedge \bar{e}_{3}, \bar{e}_{1} \wedge \bar{e}_{2} \wedge \bar{f}_{3}, \bar{e}_{1} \wedge \bar{f}_{2} \wedge \bar{e}_{3}, \bar{e}_{1} \wedge \bar{f}_{2} \wedge \bar{f}_{3}, \\
\bar{f}_{1} \wedge \bar{e}_{2} \wedge \bar{e}_{3}, \bar{f}_{1} \wedge \bar{e}_{2} \wedge \bar{f}_{3}, \bar{f}_{1} \wedge \bar{f}_{2} \wedge \bar{e}_{3}, \bar{f}_{1} \wedge \bar{f}_{2} \wedge \bar{f}_{3}, \\
\bar{e}_{1} \wedge\left(\bar{e}_{2} \wedge \bar{f}_{2}-\bar{e}_{3} \wedge \bar{f}_{3}\right), \bar{e}_{1} \wedge\left(\lambda \cdot \bar{e}_{2} \wedge \bar{f}_{2}-\lambda^{\psi} \cdot \bar{e}_{3} \wedge \bar{f}_{3}\right), \\
\bar{f}_{1} \wedge\left(\bar{e}_{2} \wedge \bar{f}_{2}-\bar{e}_{3} \wedge \bar{f}_{3}\right), \bar{f}_{1} \wedge\left(\lambda \cdot \bar{e}_{2} \wedge \bar{f}_{2}-\lambda^{\psi} \cdot \bar{e}_{3} \wedge \bar{f}_{3}\right), \\
\bar{e}_{2} \wedge\left(\bar{e}_{1} \wedge \bar{f}_{1}-\bar{e}_{3} \wedge \bar{f}_{3}\right), \bar{e}_{2} \wedge\left(\lambda \cdot \bar{e}_{1} \wedge \bar{f}_{1}-\lambda^{\psi} \cdot \bar{e}_{3} \wedge \bar{f}_{3}\right), \\
\bar{f}_{2} \wedge\left(\bar{e}_{1} \wedge \bar{f}_{1}-\bar{e}_{3} \wedge \bar{f}_{3}\right), \bar{f}_{2} \wedge\left(\lambda \cdot \bar{e}_{1} \wedge \bar{f}_{1}-\lambda^{\psi} \cdot \bar{e}_{3} \wedge \bar{f}_{3}\right), \\
\bar{e}_{3} \wedge\left(\bar{e}_{1} \wedge \bar{f}_{1}-\bar{e}_{2} \wedge \bar{f}_{2}\right), \bar{e}_{3} \wedge\left(\lambda \cdot \bar{e}_{1} \wedge \bar{f}_{1}-\lambda^{\psi} \cdot \bar{e}_{2} \wedge \bar{f}_{2}\right), \\
\bar{f}_{3} \wedge\left(\bar{e}_{1} \wedge \bar{f}_{1}-\bar{e}_{2} \wedge \bar{f}_{2}\right), \bar{f}_{3} \wedge\left(\lambda \cdot \bar{e}_{1} \wedge \bar{f}_{1}-\lambda^{\psi} \cdot \bar{e}_{2} \wedge \bar{f}_{2}\right) .
\end{gathered}
$$

We now discuss two classes of hyperplanes of $D H(5, \mathbb{K}, \psi)$.

(I) Let $\mathcal{H}$ be the hyperplane of $\operatorname{DH}(5, \mathbb{K}, \psi)$ with representative vector $\alpha=$ $\eta_{1} \cdot \bar{e}_{1} \wedge \bar{e}_{2} \wedge \bar{e}_{3}+\eta_{2} \cdot \bar{e}_{1} \wedge \bar{f}_{2} \wedge \bar{f}_{3}+\eta_{3} \cdot \bar{f}_{1} \wedge \bar{e}_{2} \wedge \bar{f}_{3}$. Let $p$ be the point $\left\langle\bar{e}_{1}, \bar{e}_{2}, \bar{f}_{3}\right\rangle$ of 
$D H(5, \mathbb{K}, \psi)$. Since $\alpha \wedge \bar{e}_{1} \wedge \bar{e}_{2} \wedge \bar{f}_{3}=0$, the point $p$ belongs to $\mathcal{H}$. An arbitrary line of $\operatorname{DH}(5, \mathbb{K}, \psi)$ through $p$ corresponds to a line $\left\langle\bar{v}_{1}, \bar{v}_{2}\right\rangle \subseteq\left\langle\bar{e}_{1}, \bar{e}_{2}, \bar{f}_{3}\right\rangle$ of $H(5, \mathbb{K}, \psi)$. Since $\left\langle\bar{v}_{1}, \bar{v}_{2}\right\rangle$ meets each of $\left\langle\bar{e}_{1}, \bar{e}_{2}\right\rangle,\left\langle\bar{e}_{1}, \bar{f}_{3}\right\rangle$ and $\left\langle\bar{e}_{2}, \bar{f}_{3}\right\rangle$, we necessarily have $\alpha \wedge \bar{v}_{1} \wedge \bar{v}_{2}=0$. So, every line of $D H(5, \mathbb{K}, \psi)$ through $p$ is contained in $\mathcal{H}$. This implies that every quad $Q$ through $p$ is either deep (i.e. $Q \subseteq \mathcal{H}$ ) or singular with deep point $p$ (i.e. $Q \cap \mathcal{H}=p^{\perp} \cap \mathcal{H}$ ).

Lemma 5.1 Let $a_{1}, a_{2}, a_{3} \in \mathbb{K}$ with $\left(a_{1}, a_{2}, a_{3}\right) \neq(0,0,0)$. The quad $Q$ through $p$ corresponding to the point $\left\langle a_{1} \bar{e}_{1}+a_{2} \bar{e}_{2}+a_{3} \bar{f}_{3}\right\rangle$ of $H(5, \mathbb{K}, \psi)$ is contained in $\mathcal{H}$ if and only if $\eta_{3} a_{1}^{\psi+1}+\eta_{2} a_{2}^{\psi+1}-\eta_{1} a_{3}^{\psi+1}=0$.

Proof. Suppose $a_{3} \neq 0$. Then $p^{\prime}=\left\langle a_{1} \bar{e}_{1}+a_{2} \bar{e}_{2}+a_{3} \bar{f}_{3}, a_{3}^{\psi} \bar{f}_{2}+a_{2}^{\psi} \bar{e}_{3}, a_{3}^{\psi} \bar{f}_{1}+\right.$ $\left.a_{1}^{\psi} \bar{e}_{3}\right\rangle$ is a point of $Q$ at distance 2 from $p$. Clearly, $Q$ is deep if and only if $p^{\prime} \in \mathcal{H}$, i.e. if and only if

$$
\begin{aligned}
\left(\eta_{1} \cdot \bar{e}_{1}\right. & \left.\wedge \bar{e}_{2} \wedge \bar{e}_{3}+\eta_{2} \cdot \bar{e}_{1} \wedge \bar{f}_{2} \wedge \bar{f}_{3}+\eta_{3} \cdot \bar{f}_{1} \wedge \bar{e}_{2} \wedge \bar{f}_{3}\right) \\
& \wedge\left(a_{1} \bar{e}_{1}+a_{2} \bar{e}_{2}+a_{3} \bar{f}_{3}\right) \wedge\left(a_{3}^{\psi} \bar{f}_{2}+a_{2}^{\psi} \bar{e}_{3}\right) \wedge\left(a_{3}^{\psi} \bar{f}_{1}+a_{1}^{\psi} \bar{e}_{3}\right)=0 .
\end{aligned}
$$

One readily verifies that this is the case if and only if $\eta_{3} a_{1}^{\psi+1}+\eta_{2} a_{2}^{\psi+1}-$ $\eta_{1} a_{3}^{\psi+1}=0$ holds in this case.

Similar calculations as above show that if $a_{1} \neq 0$ or $a_{2} \neq 0$, then $Q \subseteq \mathcal{H}$ if and only if $\eta_{3} a_{1}^{\psi+1}+\eta_{2} a_{2}^{\psi+1}-\eta_{1} a_{3}^{\psi+1}=0$.

So, the deep quads through $p$ determine a possibly degenerate Hermitian variety in the dual projective plane of $\operatorname{Res}(p)$. If the Hermitian variety $\eta_{3} X_{1}^{\psi+1}+\eta_{2} X_{2}^{\psi+1}-\eta_{1} X_{3}^{\psi+1}=0$ is empty (which is impossible in the finite case but possible in the infinite case, for instance when $\psi$ is the complex conjugation of $\mathbb{K}=\mathbb{C}$ ), then $\mathcal{H}$ is a so-called semi-singular hyperplane with deepest point $p$, i.e. $\mathcal{H}$ is of the form $p^{\perp} \cup O$, where $O$ is a set of points of $D H(5, \mathbb{K}, \psi)$ at distance 3 from $p$ such that every line at distance 2 from $p$ meets $O$ in a unique point.

(II) Recall that $H(5, \mathbb{K}, \psi)$ is the Hermitian variety of $\mathrm{PG}(5, \mathbb{K})=\mathrm{PG}(V)$ associated to $(V, f)$. With respect to the reference system $B^{*}, H(5, \mathbb{K}, \psi)$ has equation

$$
\left(X_{1} X_{2}^{\psi}-X_{2} X_{1}^{\psi}\right)+\left(X_{3} X_{4}^{\psi}-X_{4} X_{3}^{\psi}\right)+\left(X_{5} X_{6}^{\psi}-X_{6} X_{5}^{\psi}\right)=0 .
$$

Now, let $a_{1}, b_{1}, a_{2}, b_{2}, a_{3}, b_{3} \in \mathbb{K}_{0}$ and let $\omega$ be the plane of $\operatorname{PG}(5, \mathbb{K})$ with equation $X_{1}=\left(a_{1}+b_{1} \lambda\right) \cdot X_{2}, X_{3}=\left(a_{2}+b_{2} \lambda\right) \cdot X_{4}, X_{5}=\left(a_{3}+b_{3} \lambda\right) \cdot X_{6}$. Then $\omega \cap H(5, \mathbb{K}, \psi)$ is the Hermitian variety of $\omega$ with equation $b_{1} \cdot X_{2}^{\psi+1}+$ 
$b_{2} \cdot X_{4}^{\psi+1}+b_{3} \cdot X_{6}^{\psi+1}=0$. Let $X$ be the subspace of $W^{*}$ consisting of all vectors $\chi \in W^{*}$ satisfying

$$
\left(\bar{f}_{1}+\left(a_{1}+b_{1} \lambda\right) \bar{e}_{1}\right) \wedge\left(\bar{f}_{2}+\left(a_{2}+b_{2} \lambda\right) \bar{e}_{2}\right) \wedge\left(\bar{f}_{3}+\left(a_{3}+b_{3} \lambda\right) \bar{e}_{3}\right) \wedge \chi=0 .
$$

If $\left(b_{1}, b_{2}, b_{3}\right)=(0,0,0)$, then $X$ is 19 -dimensional and given by the equation

$$
\left(\bar{f}_{1}+a_{1} \bar{e}_{1}\right) \wedge\left(\bar{f}_{2}+a_{2} \bar{e}_{2}\right) \wedge\left(\bar{f}_{3}+a_{3} \bar{e}_{3}\right) \wedge \chi=0 .
$$

If $\left(b_{1}, b_{2}, b_{3}\right) \neq(0,0,0)$, then using the explicit description of the vector space $W^{*}$ given above, we see that any $\chi \in X$ also satisfies the equation

$$
\left(\bar{f}_{1}+\left(a_{1}+b_{1} \lambda^{\psi}\right) \bar{e}_{1}\right) \wedge\left(\bar{f}_{2}+\left(a_{2}+b_{2} \lambda^{\psi}\right) \bar{e}_{2}\right) \wedge\left(\bar{f}_{3}+\left(a_{3}+b_{3} \lambda^{\psi}\right) \bar{e}_{3}\right) \wedge \chi=0 .
$$

Now, for every $\eta \in \mathbb{K}^{*}$, the vector

$$
\begin{aligned}
& \chi_{\eta}:=\eta \cdot\left(\bar{f}_{1}+\left(a_{1}+b_{1} \lambda\right) \bar{e}_{1}\right) \wedge\left(\bar{f}_{2}+\left(a_{2}+b_{2} \lambda\right) \bar{e}_{2}\right) \wedge\left(\bar{f}_{3}+\left(a_{3}+b_{3} \lambda\right) \bar{e}_{3}\right) \\
& +\eta^{\psi} \cdot\left(\bar{f}_{1}+\left(a_{1}+b_{1} \lambda^{\psi}\right) \bar{e}_{1}\right) \wedge\left(\bar{f}_{2}+\left(a_{2}+b_{2} \lambda^{\psi}\right) \bar{e}_{2}\right) \wedge\left(\bar{f}_{3}+\left(a_{3}+b_{3} \lambda^{\psi}\right) \bar{e}_{3}\right) .
\end{aligned}
$$

belongs to $W^{*}$. One readily verifies that $\operatorname{dim}(X)=18$ and that the hyperplanes of $W^{*}$ with equations $\chi_{\eta} \wedge \chi=0, \eta \in \mathbb{K}^{*}$, are the $\left|\mathbb{K}_{0}\right|+1$ hyperplanes of $W^{*}$ containing $X$.

From the definition of $X$, the following is also clear: a maximal singular subspace $p$ of $H(5, \mathbb{K}, \psi)$ meets $\omega$ if and only if $e_{g r}(p) \in \operatorname{PG}(X)$. If $\left(b_{1}, b_{2}, b_{3}\right)$ can be chosen in such a way that the Hermitian variety $b_{1} X_{2}^{\psi+1}+b_{2} X_{4}^{\psi+1}+$ $b_{3} X_{6}^{\psi+1}=0$ of $\omega$ is empty, then $\operatorname{PG}(X) \cap e_{g r}(\mathcal{P})=\emptyset$, where $\mathcal{P}$ denotes the point-set of $\operatorname{DH}(5, \mathbb{K}, \psi)$. (Again, this is impossible in the finite case, but possible when $\psi$ is the complex conjugation of $\mathbb{K}=\mathbb{C}$.) This implies that every hyperplane of $\mathrm{DH}(5, \mathbb{K}, \psi)$ arising from a hyperplane of $\mathrm{PG}\left(W^{*}\right)$ through $\mathrm{PG}(X)$ cannot contain lines. Each such hyperplane is a so-called ovoid of $\operatorname{DH}(5, \mathbb{K}, \psi)$, i.e. a set of points of $D H(5, \mathbb{K}, \psi)$ meeting each line in a unique point.

\subsection{The hyperplanes of $D H\left(5, q^{2}\right)$ arising from the Grass- mann embedding}

In this section, we suppose that $n=3, \mathbb{K}=\mathbb{F}_{q^{2}}$ and $\mathbb{K}_{0}=\mathbb{F}_{q}$. Then $x^{\psi}=x^{q}$ for every $x \in \mathbb{F}_{q^{2}}$. Let $\mathcal{P}$ denote the point set of $D H\left(5, q^{2}\right)$, let $B^{*}=$ $\left(\bar{e}_{1}, \bar{f}_{1}, \bar{e}_{2}, \bar{f}_{2}, \bar{e}_{3}, \bar{f}_{3}\right)$ be a given hyperbolic basis of $V$, let $W^{*}=W\left(B^{*}\right)$ and let $e_{g r}$ denote the Grassmann embedding of $D H\left(5, q^{2}\right)$ in $\mathrm{PG}\left(W^{*}\right)$. Every quad of $D H\left(5, q^{2}\right)$ is isomorphic to $Q^{-}(5, q)$. The generalized quadrangle $Q^{-}(5, q)$ admits subquadrangles isomorphic to $Q(4, q)$, see Payne and Thas [10]. For 
any two hyperplanes $\mathcal{H}_{1}$ and $\mathcal{H}_{2}$ of $D H\left(5, q^{2}\right)$ arising from $e_{g r}$, let $\left[\left[\mathcal{H}_{1}, \mathcal{H}_{2}\right]\right]$ denote the set of all hyperplanes of $D H\left(5, q^{2}\right)$ of the form $e_{g r}^{-1}\left(e_{g r}(\mathcal{P}) \cap \pi\right)$, where $\pi$ is one of the $q+1$ hyperplanes of $\operatorname{PG}\left(W^{*}\right)$ containing $\left\langle e_{g r}\left(\mathcal{H}_{1}\right)\right\rangle \cap$ $\left\langle e_{g r}\left(\mathcal{H}_{2}\right)\right\rangle$. If $\alpha_{i} \in W^{*}, i \in\{1,2\}$, is a representative vector of $\mathcal{H}_{i}$, then the representative vectors of the hyperplanes of $\left[\left[\mathcal{H}_{1}, \mathcal{H}_{2}\right]\right]$ are precisely the vectors $\lambda_{1} \alpha_{1}+\lambda_{2} \alpha_{2}$, where $\lambda_{1}, \lambda_{2} \in \mathbb{F}_{q}$ with $\left(\lambda_{1}, \lambda_{2}\right) \neq(0,0)$.

By De Bruyn and Pralle [9], $D H\left(5, q^{2}\right)$ has 5 isomorphism classes of hyperplanes which arise from $e_{g r}$. We now give a description and a representative vector of a hyperplane of each of these classes.

(I) The hyperplanes of Type $I$ of $D H\left(5, q^{2}\right)$ are the so-called singular hyperplanes. If $x$ is a point of $D H\left(5, q^{2}\right)$, then the set $\mathcal{H}_{x}$ of points of $\operatorname{DH}\left(5, q^{2}\right)$ at distance at most 2 from $x$ is a hyperplane of $D H\left(5, q^{2}\right)$, the so-called singular hyperplane of $\mathrm{DH}\left(5, q^{2}\right)$ with deepest point $x$. If $x$ coincides with the point $\left\langle\bar{e}_{1}, \bar{e}_{2}, \bar{e}_{3}\right\rangle$, then $\bar{e}_{1} \wedge \bar{e}_{2} \wedge \bar{e}_{3}$ is a representative vector of $\mathcal{H}_{x}$.

(II) The hyperplanes of Type II of $D H\left(5, q^{2}\right)$ are the so-called extensions of the $Q(4, q)$-subquadrangles of the quads. If $\rho$ is a $Q(4, q)$-subquadrangle of a quad $Q$, then the set $\mathcal{H}_{\rho}$ of points at distance at most 1 from $\rho$ is a hyperplane of $D H\left(5, q^{2}\right)$, the so-called extension of $\rho$. By De Bruyn and Pralle [9], if $x_{1}$ and $x_{2}$ are two points of $D H\left(5, q^{2}\right)$ at distance 2 from each other, then every hyperplane of $\left[\left[\mathcal{H}_{x_{1}}, \mathcal{H}_{x_{2}}\right]\right] \backslash\left\{\mathcal{H}_{x_{1}}, \mathcal{H}_{x_{2}}\right\}$ is the extension of a $Q(4, q)$ subquadrangle of the quad $\left\langle x_{1}, x_{2}\right\rangle$. If $x_{1}=\left\langle\bar{e}_{1}, \bar{e}_{2}, \bar{e}_{3}\right\rangle$ and $x_{2}=\left\langle\bar{e}_{1}, \bar{f}_{2}, \bar{f}_{3}\right\rangle$, then $\bar{e}_{1} \wedge \bar{e}_{2} \wedge \bar{e}_{3}+\bar{e}_{1} \wedge \bar{f}_{2} \wedge \bar{f}_{3}$ is a representative vector of a hyperplane of the set $\left[\left[\mathcal{H}_{x_{1}}, \mathcal{H}_{x_{2}}\right]\right] \backslash\left\{\mathcal{H}_{x_{1}}, \mathcal{H}_{x_{2}}\right\}$.

(III) A hyperplane of $D H\left(5, q^{2}\right)$ is said to be of Type III if it belongs to some set $\left[\left[\mathcal{H}_{x_{1}}, \mathcal{H}_{x_{2}}\right]\right] \backslash\left\{\mathcal{H}_{x_{1}}, \mathcal{H}_{x_{2}}\right\}$ where $x_{1}$ and $x_{2}$ are two points of $\operatorname{DH}\left(5, q^{2}\right)$ at distance 3 from each other. The vector $\bar{e}_{1} \wedge \bar{e}_{2} \wedge \bar{e}_{3}+\bar{f}_{1} \wedge \bar{f}_{2} \wedge \bar{f}_{3}$ is a representative vector of such a hyperplane.

(IV) A hyperplane of $D H\left(5, q^{2}\right)$ is said to be of Type $I V$ if it belongs to some set $\left[\left[\mathcal{H}_{\rho_{1}}, \mathcal{H}_{\rho_{2}}\right]\right] \backslash\left\{\mathcal{H}_{\rho_{1}}, \mathcal{H}_{\rho_{2}}\right\}$ where (i) $\rho_{i}, i \in\{1,2\}$, is a $Q(4, q)$-subquadrangle of a quad $Q_{i}$ of $D H\left(5, q^{2}\right)$, (ii) $Q_{1} \cap Q_{2}$ is a line $L$, (iii) $L \subseteq \rho_{1}$ and $\left|\rho_{2} \cap L\right|=$ 1 (see [9, Section 4.5]). By De Bruyn and Pralle [9], a hyperplane $\mathcal{H}$ of $D H\left(5, q^{2}\right)$ is of type IV if and only if there exists a (necessarily unique) point $x$ such that (i) $x^{\perp} \subseteq \mathcal{H}$ and (ii) the set of deep quads through $x$ is a nondegenerate Hermitian curve in the dual projective plane of Res $(x)$. By Lemma 5.1, the vector $\bar{e}_{1} \wedge \bar{e}_{2} \wedge \bar{e}_{3}+\bar{e}_{1} \wedge \bar{f}_{2} \wedge \bar{f}_{3}+\bar{f}_{1} \wedge \bar{e}_{2} \wedge \bar{f}_{3}$ is a representative vector of a hyperplane of Type IV.

(V) With respect to the reference system $B^{*}, H\left(5, q^{2}\right)$ has the equation $\left(X_{1} X_{2}^{q}-X_{2} X_{1}^{q}\right)+\left(X_{3} X_{4}^{q}-X_{4} X_{3}^{q}\right)+\left(X_{5} X_{6}^{q}-X_{6} X_{5}^{q}\right)=0$. Let $\omega$ be a 
plane of $\mathrm{PG}\left(5, q^{2}\right)$ which intersects $H\left(5, q^{2}\right)$ in a unital of $\omega$ and let $S_{\omega}$ be the set of planes of $H\left(5, q^{2}\right)$ meeting $\omega$. By De Bruyn and Pralle [9, Corollary 4.29], $\left\langle e_{g r}\left(S_{\omega}\right)\right\rangle$ is a 17-dimensional subspace of $\mathrm{PG}\left(W^{*}\right)$. A hyperplane of $D H\left(5, q^{2}\right)$ is said to be of Type $V$ if it is isomorphic to some hyperplane of the form $e_{g r}^{-1}\left(e_{g r}(\mathcal{P}) \cap \pi\right)$, where $\pi$ is one of the $q+1$ hyperplanes of $\operatorname{PG}\left(W^{*}\right)$ containing $\left\langle e_{g r}\left(S_{\omega}\right)\right\rangle$.

Now, for every $\lambda \in \mathbb{F}_{q^{2}} \backslash \mathbb{F}_{q}$, let $\omega_{\lambda}$ be the plane of $\operatorname{PG}\left(5, q^{2}\right)$ with equation $X_{1}=\lambda \cdot X_{2}, X_{3}=\lambda \cdot X_{4}, X_{5}=\lambda \cdot X_{6}$. Then $\omega_{\lambda} \cap H\left(5, q^{2}\right)$ is a unital of $\omega_{\lambda}$. The 17-dimensional subspace $\left\langle e_{g r}\left(S_{\omega_{\lambda}}\right)\right\rangle$ of $\mathrm{PG}\left(W^{*}\right)$ consists of all points $\langle\chi\rangle$ of $\operatorname{PG}\left(W^{*}\right)$, where $\chi$ is a nonzero vector of $W^{*}$ satisfying

$$
\left(\bar{f}_{1}+\lambda \bar{e}_{1}\right) \wedge\left(\bar{f}_{2}+\lambda \bar{e}_{2}\right) \wedge\left(\bar{f}_{3}+\lambda \bar{e}_{3}\right) \wedge \chi=0 .
$$

Now, for every $\eta \in \mathbb{F}_{q^{2}}^{*}, \chi_{\lambda, \eta}:=\eta \cdot\left(\bar{f}_{1}+\lambda \bar{e}_{1}\right) \wedge\left(\bar{f}_{2}+\lambda \bar{e}_{2}\right) \wedge\left(\bar{f}_{3}+\lambda \bar{e}_{3}\right)+\eta^{q}$. $\left(\bar{f}_{1}+\lambda^{q} \bar{e}_{1}\right) \wedge\left(\bar{f}_{2}+\lambda^{q} \bar{e}_{2}\right) \wedge\left(\bar{f}_{3}+\lambda^{q} \bar{e}_{3}\right)$ is a vector of $W^{*}$ and the equations $\chi_{\lambda, \eta} \wedge \chi=0, \eta \in \mathbb{F}_{q^{2}}^{*}$, determine the $q+1$ hyperplanes of $\mathrm{PG}\left(W^{*}\right)$ containing $\left\langle e_{g r}\left(S_{\omega_{\lambda}}\right)\right\rangle$, see Section 5.1 (II). So, for any $\lambda \in \mathbb{F}_{q^{2}} \backslash \mathbb{F}_{q}$ and any $\eta \in \mathbb{F}_{q^{2}}^{*}, \chi_{\lambda, \eta}$ is a representative vector of a hyperplane of Type V. Our aim is now to give a representative vector of a nicer form.

Let $\eta_{1}, \eta_{2} \in \mathbb{F}_{q}^{*}$ such that the polynomial $\eta_{2} X^{2}+\left(\eta_{1} \eta_{2}+\eta_{1}+\eta_{2}\right) X+\eta_{1} \in$ $\mathbb{F}_{q}[X]$ is irreducible. Such a polynomial exists by the following lemma.

Lemma 5.2 For every irreducible monic quadratic polynomial $X^{2}+a X+b \in$ $\mathbb{F}_{q}[X]$, there exist unique elements $\eta_{1}, \eta_{2} \in \mathbb{F}_{q} \backslash\{0\}$ such that $\eta_{2}\left(X^{2}+a X+b\right)=$ $\eta_{2} X^{2}+\left(\eta_{1} \eta_{2}+\eta_{1}+\eta_{2}\right) X+\eta_{1}$.

Proof. Since $X^{2}+a X+b$ is irreducible, its values at the points -1 and 0 are nonzero. Hence, $b \neq 0$ and $a-1-b \neq 0$. After an easy and straightforward computation, we find that there is only one solution for $\eta_{1}$ and $\eta_{2}$, namely $\eta_{1}=a-1-b$ and $\eta_{2}=\frac{a-1-b}{b}$.

Suppose now that $\lambda \in \mathbb{F}_{q^{2}} \backslash \mathbb{F}_{q}$ is a root of the polynomial $\eta_{2} X^{2}-\left(\eta_{1} \eta_{2}+\right.$ $\left.\eta_{1}+\eta_{2}\right) X+\eta_{1}$ and $\eta=\frac{\lambda^{q}-1}{\lambda\left(\lambda^{q}-\lambda\right)}$. Then $\lambda+\lambda^{q}=\frac{\eta_{1}+\eta_{2}+\eta_{1} \eta_{2}}{\eta_{2}}$ and $\lambda^{q+1}=\frac{\eta_{1}}{\eta_{2}}$. One calculates that $\chi_{\lambda, \eta}=\eta_{1} \cdot \bar{e}_{1} \wedge \bar{e}_{2} \wedge \bar{e}_{3}+\eta_{2} \cdot \bar{f}_{1} \wedge \bar{f}_{2} \wedge \bar{f}_{3}+\left(\bar{e}_{1}+\bar{f}_{1}\right) \wedge\left(\bar{e}_{2}+\right.$ $\left.\bar{f}_{2}\right) \wedge\left(\bar{e}_{3}+\bar{f}_{3}\right)=0$.

Let $\mathcal{A}$ denote the group of automorphisms of $D H\left(5, q^{2}\right)$. By De Bruyn and Pralle [9], $\mathcal{A}$ has 5 orbits on the set of hyperplanes of $D H\left(5, q^{2}\right)$ arising from $e_{g r}$. [A more careful inspection of the proof of [9] would reveal that there are still five orbits if we restrict to those automorphisms which arise from projectivities of $\operatorname{PG}\left(5, q^{2}\right)=\operatorname{PG}(V)$.] For every $\varphi \in \mathcal{A}$, there exists a unique projectivity $\widetilde{\varphi}$ of $\mathrm{PG}\left(W^{*}\right)$ such that $e_{g r}(\varphi(p))=\widetilde{\varphi}\left(e_{g r}(p)\right)$ for every point 
$p$ of $D H\left(5, q^{2}\right)$. In view of the bijective correspondence between the set of hyperplanes of $\mathrm{DH}\left(5, q^{2}\right)$, the set of hyperplanes of $\mathrm{PG}\left(W^{*}\right)$ and the set of points of $\mathrm{PG}\left(W^{*}\right)$ (use the polarity $\zeta^{*}$ defined in Section 4), the group $\widetilde{A}:=\{\widetilde{\varphi} \mid \varphi \in \mathcal{A}\}$ has 5 orbits on the set of hyperplanes of $\mathrm{PG}\left(W^{*}\right)$ and also 5 orbits on the set of points of $\mathrm{PG}\left(W^{*}\right)$. Representatives of these 5 orbits are the points $\left\langle\bar{e}_{1} \wedge \bar{e}_{2} \wedge \bar{e}_{3}\right\rangle,\left\langle\bar{e}_{1} \wedge \bar{e}_{2} \wedge \bar{e}_{3}+\bar{e}_{1} \wedge \bar{f}_{2} \wedge \bar{f}_{3}\right\rangle,\left\langle\bar{e}_{1} \wedge \bar{e}_{2} \wedge \bar{e}_{3}+\bar{f}_{1} \wedge \bar{f}_{2} \wedge \bar{f}_{3}\right\rangle$, $\left\langle\bar{e}_{1} \wedge \bar{e}_{2} \wedge \bar{e}_{3}+\bar{e}_{1} \wedge \bar{f}_{2} \wedge \bar{f}_{3}+\bar{f}_{1} \wedge \bar{e}_{2} \wedge \bar{f}_{3}\right\rangle$ and $\left\langle\eta_{1} \cdot \bar{e}_{1} \wedge \bar{e}_{2} \wedge \bar{e}_{3}+\eta_{2} \cdot \bar{f}_{1} \wedge \bar{f}_{2} \wedge\right.$ $\left.\bar{f}_{3}+\left(\bar{e}_{1}+\bar{f}_{1}\right) \wedge\left(\bar{e}_{2}+\bar{f}_{2}\right) \wedge\left(\bar{e}_{3}+\bar{f}_{3}\right)\right\rangle$ (with $\eta_{1}$ and $\eta_{2}$ as above).

\section{References}

[1] R. J. Blok, I. Cardinali, B. De Bruyn and A. Pasini. Polarized and homogeneous embeddings of dual polar spaces. J. Algebraic Combin., to appear.

[2] R. J. Blok and B. N. Cooperstein. The generating rank of the unitary and symplectic grassmannians. preprint.

[3] P. J. Cameron. Dual polar spaces. Geom. Dedicata 12 (1982), 75-85.

[4] I. Cardinali, B. De Bruyn and A. Pasini. Minimal full polarized embeddings of dual polar spaces. J. Algebraic Combin. 25 (2007), 7-23.

[5] B. N. Cooperstein. On the generation of dual polar spaces of unitary type over finite fields. European J. Combin. 18 (1997), 849-856.

[6] B. De Bruyn. Near polygons. Frontiers in Mathematics. Birkhäuser Verlag, Basel, 2006.

[7] B. De Bruyn. On the Grassmann-embeddings of the hermitian dual polar spaces. Linear Multilinear Algebra 56 (2008), 665-677.

[8] B. De Bruyn. On the Grassmann modules for the symplectic groups. Preprint.

[9] B. De Bruyn and H. Pralle. The hyperplanes of $D H\left(5, q^{2}\right)$. Forum Math. 20 (2008), 239-264.

[10] S. E. Payne and J. A. Thas. Finite Generalized Quadrangles. Research Notes in Mathematics 110. Pitman, Boston, 1984.

[11] E. E. Shult. On Veldkamp lines. Bull. Belg. Math. Soc. Simon Stevin 4 (1997), 299-316. 
[12] J. Tits. Buildings of spherical type and finite BN-pairs. Lecture Notes in Mathematics 386. Springer, Berlin, 1974.

[13] F. D. Veldkamp. Polar Geometry. I-IV, V. Indag. Math. 21 and 22 (1959), 512-551 and 207-212. 